\title{
ON THE MODULUS OF MEASURES WITH VALUES IN TOPOLOGICAL RIESZ SPACES
}

\author{
Lech DREWNOWSKI and Witold WNUK*
}

\begin{abstract}
The paper is devoted to a study of some aspects of the theory of (topological) Riesz space valued measures. The main topics considered are the following. First, the problem of existence (and, particularly, the so-called proper existence) of the modulus of an order bounded measure, and its relation to a similar problem for the induced integral operator. Second, the question of how properties of such a measure like countable additivity, exhaustivity or socalled absolute exhaustivity, or the properties of the range space, influence the properties of the modulus of the measure. Third, the problem of exhibiting (or constructing) Banach lattices that are "good" in many respects, and yet admit a countably additive measure whose modulus is not countably additive. A few applications to weakly compact operators from spaces of bounded measurable functions to Banach lattices are also presented.
\end{abstract}

\section{Introduction}

Let $\mu$ be a countably additive and order bounded measure from a $\sigma$ algebra $\Sigma$ to a Banach lattice $E$. If the modulus $|\mu|$ of $\mu$ exists, it is natural to ask whether it must be countably additive as well. This problem has been recently considered by H. H. Schaefer and X. D. Zhang [hSZ], and G. Kronsbein and P. Meyer-Nieberg [KM-N]. They proved that it is so when $E$ has order continuous norm or is a Dedekind complete AM-space with a strong unit [hSZ] (see also [kS2]), or if $E$ contains no

\footnotetext{
*The research of both authors was supported in part by Komitet Badań Naukowych (State Committee for Scientific Research), Poland, grant no 2 P301 003 07.
}

2000 Mathematics Subject Classification: Primary 46B42, 46G10.

Servicio de Publicaciones. Universidad Complutense. Madrid, 2002 
isomorphic copy of $\ell_{\infty}[\mathrm{KM}-\mathrm{N}]$. (For some related earlier results, see [kS1], [kS2], and [kS3].) A particular case of this problem for the moduli of indefinite Bochner or Pettis integrals of strongly measurable functions has been dealt with by the present authors in [DW]. Therein, an example was also given showing that, in general, the above problem has a negative answer even if $E$ is Dedekind complete (which forces the existence of $|\mu|$ ). We continue our investigations in the present paper. This time, however, we work in the setting of general locally solid (topological) Riesz spaces (TRS), and we include also exhaustive finitely additive measures in the area of our research. A brief description of the main points of the paper follows.

In Section 1, we collect some preliminary results concerning absolute and positive majorants and the notion of the modulus for a Riesz space valued measure. Next, in Section 2, we construct an order bounded $\sigma$ additive measure with values in the Banach lattice $c$ that has no absolute majorant. (Another example to the same effect is given in Section 7.)

As for Section 3, assume for simplicity that $E$ and $\mu$ are as in the beginning. Then we associate with the measure $\mu$ its absolute semivariation $\|\mu\|_{a}: \Sigma \rightarrow \overline{\mathbb{R}}_{+}$defined by

$$
\|\mu\|_{a}(A)=\sup \left\|\left|\mu\left(B_{1}\right)\right|+\cdots+\left|\mu\left(B_{n}\right)\right|\right\|,
$$

where the sup is taken over all $\Sigma$-partitions $B_{1}, \ldots, B_{n}$ of $A$. We prove that $\|\mu\|_{a}$ is order continuous (or exhaustive) iff $\mu$ is absolutely exhaustive, i.e., the series $\sum_{n}\left|\mu\left(C_{n}\right)\right|$ converges in norm for every disjoint sequence $\left(C_{n}\right)$ in $\Sigma$. A condition of exactly the latter type was already shown in [DW, Th. 2] to characterize those indefinite Pettis integrals of strongly measurable functions whose modulus exists and is $\sigma$-additive.

Motivated by this, we introduce in Section 4 a new class of TRS's as follows: We say that a TRS $E$ has Property $(S)$ if for every subseries convergent series $\sum_{n} x_{n}$ in $E$ with order bounded sums $\sum_{i=1}^{n}\left|x_{i}\right|$ also the series $\sum_{n}\left|x_{n}\right|$ is convergent. For example, all Lebesgue (or order continuous) Banach lattices and all AM-spaces have Property (S). This property is obviously inherited by arbitrary products and sequentially closed sublattices of TRS's having (S). We also consider Property (CS) which is a Cauchy variant of Property (S). As we have learned at a later stage of working on this paper, the class of Banach lattices satisfying the above condition has already been isolated in [G, Def. 4.3, p. 94] and called Banach lattices with property $(\mathrm{C})$. In a later paper [GvR], such 
Banach lattices are said to be order bounded $A M$.

In Section 5, we first characterize TRS's that are $\sigma$-Dedekind complete and $\sigma$-Lebesgue as those satisfying a mild completeness condition and containing no positive copy of the Banach lattice $c$. We next recall from [DL] a useful characterization of TRS's containing no positive copy of the Banach lattice $\ell_{\infty}$.

In Section 6, we use our results concerning absolute semivariations and Property $(\mathrm{S})$, as well as some more direct results relating the exhaustivity of $|\mu|$ to its $\sigma$-additivity, to give far going generalizations (with much simpler proofs) of the results from [hSZ] and [KM-N] quoted above.

In Section 7, we strengthen our negative answer to the question of Schaefer and Zhang by showing that if a $\sigma$-Dedekind complete Banach lattice $E$ is atomless and the ideal $E_{A}$ of its order continuous elements is not a band, then one can find an order bounded $\sigma$-additive (and even atomless) $E$-valued measure whose modulus exists but is not $\sigma$-additive. We also give, following [G], an example of an order bounded $\sigma$-additive measure $\mu$ with values in a Dedekind complete AM-space for which $|\mu|$ is not $\sigma$-additive.

In Section 8, we collect some results concerning the so called proper existence of the modulus of a measure $\mu$, and establish a close relationship between this and an analogous problem for the induced integral operator $I_{\mu}$.

Finally, Section 9 contains a few results dealing with absolute exhaustivity and weak compactness of integral operators of type $I_{\mu}$.

Acknowledgment. We are extremely grateful to Professor Zbigniew Lipecki for many helpful and stimulating comments and suggestions concerning an earlier draft of this paper. (In particular, Proposition 1.5 is due to him.) We also thank him very much for calling our attention to several previous works that are closely related to the subject matter of our paper and, particularly, for pointing out the dissertation of G. Groenewegen $[\mathrm{G}]$ and providing us with its copy. 


\section{Absolute majorants and the modulus of a mea- sure}

Here we collect some basic terminology and notation along with a few simple, mostly "folklore", facts. We refer the reader to [AB1], [AB2], and $[\mathrm{hS}]$ for general Riesz spaces (or vector lattices), locally solid (topological) Riesz spaces and Banach lattices, and to [DU] and [DS] for vector measures and integrals.

Throughout, $\mathcal{A}$ is an algebra, and $\Sigma$ is a $\sigma$-algebra, of subsets of a set $S$. (Obviously, the definitions and results involving $\mathcal{A}$ apply in particular to $\Sigma$.) Depending on the context, all subsets of $S$ occurring below are tacitly assumed to be members of $\mathcal{A}$ or $\Sigma$; a partition of a set $A \subset S$ means a finite $\mathcal{A}$ - or $\Sigma$-partition of $A$; and $\Pi(A)$ stands for the family of all such partitions of $A$ (directed upward by refinement). If $B \in \mathcal{A}$ and $\mathcal{C} \subset \mathcal{A}$, then

$$
\mathcal{C}_{B} \equiv \mathcal{C} \cap B:=\{C \cap B: C \in \mathcal{C}\} .
$$

In particular, $\mathcal{A}_{B}=\{A \in \mathcal{A}: A \subset B\}$ is the algebra of subsets of $B$ induced by $\mathcal{A}$. Likewise, for $B \in \Sigma, \Sigma_{B}$ is the induced $\sigma$-algebra on $B$. The characteristic function of a set $A \subset S$ is denoted $1_{A}$. Finally, $\mathbb{N}=\{1,2, \ldots\}$, and $\mathcal{P}(\mathbb{N})$ denotes the power set of $\mathbb{N}$.

We use the abbreviation TVs for Hausdorff topological vector space, and TRs for Hausdorff locally solid (topological) Riesz space. We follow [AB1] and [AB2] quite closely in terminology concerning TRS's. We denote by $\omega$ the Riesz space of all real sequences (with the product topology), and by $\ell_{\infty}, c$, and $c_{0}$ the standard Banach lattices of real sequences that are, respectively, bounded, convergent, or convergent to zero (equipped with the sup norm). As usual, $\left(e_{n}\right)$ will stand for the unit vectors in these (and other) sequence spaces.

By a measure we always mean a finitely additive measure (defined on $\mathcal{A}$ or $\Sigma$ ), and the requirement of countable (or $\sigma$-) additivity will always be imposed explicitly when needed.

Recall that a measure $\mu$ defined on $\mathcal{A}$ and taking values in a TVs $E$ is said to be exhaustive (or strongly additive [DU]) if $\mu\left(D_{n}\right) \rightarrow 0$ for every disjoint sequence $\left(D_{n}\right)$ in $\mathcal{A}$ or, equivalently, if the series $\sum_{n} \mu\left(D_{n}\right)$ is (unconditionally or subseries) Cauchy for every disjoint sequence $\left(D_{n}\right)$ in $\mathcal{A}$. Another equivalent reformulation is that the sequence $\left(\mu\left(A_{n}\right)\right)$ has 
to be Cauchy whenever $A_{n} \uparrow$ in $\mathcal{A}$. Clearly, "Cauchy" can be replaced by "convergent" in the above conditions when $E$ is sequentially complete, or when $E$ is a TRS with the $\sigma$-monotone completeness property for intervals (see Section 4 ) and the measure $\mu$ assumes only positive values $(\mu \geqslant 0)$.

Also recall that a submeasure on $\mathcal{A}$ is a function $\eta: \mathcal{A} \rightarrow \overline{\mathbb{R}}_{+}$that is nondecreasing, subadditive, and vanishes at $\emptyset$. It is said to be exhaustive if $\eta\left(D_{n}\right) \rightarrow 0$ for every disjoint sequence $\left(D_{n}\right)$ in $\mathcal{A}$; order continuous if $\eta\left(A_{n}\right) \rightarrow 0$ whenever $\left(A_{n}\right) \subset \mathcal{A}$ and $A_{n} \downarrow \emptyset$. Note that an analogous property of order continuity for measures taking values in a TVS coincides with $\sigma$-additivity.

Let $E$ be a Riesz space, and let $\mu, \nu: \mathcal{A} \rightarrow E$ be measures. We write $\mu \leqslant \nu$ if $\mu(A) \leqslant \nu(A)$ for all $A \in \mathcal{A}$. By an absolute (resp., positive) majorant for $\mu$ we mean a measure $\gamma: \mathcal{A} \rightarrow E_{+}$such that $|\mu(A)| \leqslant \gamma(A)$ (resp., $\mu(A) \leqslant \gamma(A)$ ) for all $A \in \mathcal{A}$. The smallest absolute (resp., positive) majorant for $\mu$, if exists, is called the modulus (resp., positive part) of $\mu$ and denoted $|\mu|$ (resp., $\mu^{+}$). It is not hard to see that $\mu$ admits an absolute majorant iff it admits a positive majorant, and that $|\mu|$ exists iff $\mu^{+}$exists; see Proposition 1.4 below for more details. An obvious condition that is necessary (though not sufficient, see Section 2) for the existence of an absolute or positive majorant for $\mu$ is that $\mu$ is order bounded, i.e., that its range $\mu(\mathcal{A})$ is order bounded. In view of the equivalencies mentioned above, in what follows we focus our attention on the existence and properties of $|\mu|$. Note that if $|\mu|$ exists, then $|\mu(A)| \leqslant|\mu|(A)$ for each $A \in \mathcal{A}$, whence

$$
\sum_{D \in \pi}|\mu(D)| \leqslant|\mu|(A) \quad \text { for all } A \in \mathcal{A} \text { and } \pi \in \Pi(A) .
$$

To shorten formulations, we adopt the convention that a phrase like "assume that the modulus $|\mu|$ exists" is an abbreviation for "assume that $\mu$ is order bounded and that the modulus $|\mu|$ exists".

Proposition 1.1. If the modulus $|\mu|$ of a measure $\mu: \mathcal{A} \rightarrow E$ exists, then for every $B \in \mathcal{A}$ the measure $\mu_{B}=\mu \mid \mathcal{A}_{B}$ has a modulus, and $\left|\mu_{B}\right|=|\mu| \mid \mathcal{A}_{B}$.

Proof. Clearly, $|\mu|_{B}=|\mu| \mid \mathcal{A}_{B}$ is an absolute majorant for $\mu_{B}$. Let a measure $\gamma: \mathcal{A}_{B} \rightarrow E$ be an absolute majorant for $\mu_{B}$. Then the measure $\nu: \mathcal{A} \rightarrow E$ defined by $\nu(A)=\gamma(A \cap B)+|\mu|(A \backslash B)$ is an absolute 
majorant for $\mu$, hence $|\mu| \leqslant \nu$. In consequence, $|\mu|_{B} \leqslant \nu \mid \mathcal{A}_{B}=\gamma$, and it follows that $|\mu|_{B}$ is the smallest absolute majorant for $\mu_{B}$.

We now recall a useful general fact, where the first (basic) equality follows by an easy induction from the identity $|x|+|y|=|x+y| \vee|x-y|$ (cf. [hS, p. 52, Cor. 1 (8)]).

Fact. For any finite family $\left\{x_{j}: j \in J\right\}$ in a Riesz space $E$,

$$
\begin{aligned}
\sum_{j \in J}\left|x_{j}\right| & =\sup \left\{\left|\sum_{j \in J} \varepsilon_{j} x_{j}\right|: \varepsilon_{j}= \pm 1\right\}=\sup \left\{\sum_{j \in K} x_{j}-\sum_{j \in J \backslash K} x_{j}: K \subset J\right\} \\
& =\sup \left\{\sum_{j \in K} x_{j}-\sum_{j \in L} x_{j}: K, L \subset J\right\} .
\end{aligned}
$$

Hence, if $\left|\sum_{j \in K} x_{j}\right| \leqslant x$ for all $K \subset J$ and some $x \in E_{+}$, then $\sum_{j \in J}\left|x_{j}\right| \leqslant 2 x$.

From this the following is easily obtained (cf. [DW], [G, p. 83], [kS2, 4. Remarks]).

Proposition 1.2. Let $\mu: \mathcal{A} \rightarrow E$ be a measure. Then for any finite disjoint family $\left\{B_{j}: j \in J\right\}$ in $\mathcal{A}$,

$$
\sum_{j \in J}\left|\mu\left(B_{j}\right)\right|=\sup \left\{\mu\left(\bigcup_{j \in K} B_{j}\right)-\mu\left(\bigcup_{j \in L} B_{j}\right): K, L \subset J\right\} .
$$

In consequence, for every $A \in \mathcal{A}$,

(a) if one of the sups below exists, so do the other and they are all equal:

$$
\begin{aligned}
\sup \left\{\sum_{D \in \pi}|\mu(D)|: \pi \in \Pi(A)\right\} & =\sup \{\mu(B)-\mu(C): B, C \subset A\} \\
& =\sup \{\mu(B)-\mu(A \backslash B): B \subset A\} .
\end{aligned}
$$

(b) if $\mu$ is order bounded, so is the increasing net $\left\{\sum_{D \in \pi}|\mu(D)|\right.$ : $\pi \in \Pi(A)\}$.

Corollary 1.3. Let $\mu: \mathcal{A} \rightarrow E$ be an order bounded measure. Then for every disjoint sequence $\left(D_{n}\right)$ in $\mathcal{A}$, the series $\sum_{n}\left|\mu\left(D_{n}\right)\right|$ is order bounded; that is, the sequence of its partial sums is order bounded. 
It is easily seen that if $E$ is Dedekind complete and $\mu: \mathcal{A} \rightarrow E$ is an order bounded measure, then the modulus $|\mu|$ exists and is given by each of the equalities below:

$$
\begin{aligned}
|\mu|(A) & =\sup \left\{\sum_{D \in \pi}|\mu(D)|: \pi \in \Pi(A)\right\} \\
& =\sup \{\mu(B)-\mu(A \backslash B): B \subset A\} \\
& =\sup \{\mu(B)-\mu(C): B, C \subset A\} \\
& =\sup \{|\mu(B)-\mu(C)|: B, C \subset A\} .
\end{aligned}
$$

Moreover, it is also easily verified that whenever the above formulas make sense for each $A \in \mathcal{A}$, they define a positive measure, and it is precisely the modulus $|\mu|$ of $\mu$. When this is the case, we shall say (following [DW]) that the modulus $|\mu|$ exists properly, or that $\mu$ has a proper modulus. Also, when the modulus $|\mu|$ exists, we shall say that it exists properly on a set $A \in \mathcal{A}$ if $|\mu|(A)$ is given by the above formulas.

It is apparently an open question whether an order bounded measure may have a non-proper modulus. At the end of the paper (Section 8), we give a few results that are relevant to this question. Here we only state the following proposition. Its parts (a) and (b) follow from the fact that the formula $\gamma=2 \nu-\mu$ establishes an order isomorphism between positive majorants $\nu$ and absolute majorants $\gamma$ for the measure $\mu$, while part (c) follows directly from the equality $\mu(B)=\frac{1}{2}(\mu(B)-\mu(A \backslash B))+$ $\frac{1}{2} \mu(A)$ for $B \subset A \in \mathcal{A}$.

Proposition 1.4. Let $\mu: \mathcal{A} \rightarrow E$ be an order bounded measure.

(a) $\mu$ admits an absolute majorant iff $\mu$ admits a positive majorant.

(b) $|\mu|$ exists iff $\mu^{+}$exists, and if this is the case, then $\mu^{+}=\frac{1}{2}(|\mu|+\mu)$ and, consequently, $\mu=\mu^{+}-\mu^{-}$and $|\mu|=\mu^{+}+\mu^{-}$, where $\mu^{-}:=$ $(-\mu)^{+}$.

(c) $|\mu|$ exists properly iff $\mu^{+}(A)=\sup \{\mu(B): B \subset A\}$ in $E$ for every $A \in \mathcal{A}$.

Before proceeding let us recall that a Fréchet-Nikodym topology on $\mathcal{A}$ is one for which the operations of symmetric difference $(A, B) \rightarrow A \triangle B$ 
and intersection $(A, B) \rightarrow A \cap B$ are uniformly continuous. Equivalently, it is a topology converting $(\mathcal{A}, \triangle)$ to a topological abelian group with a base of neighborhoods of $\emptyset$ consisting of solid classes $\mathcal{U} \subset \mathcal{A}$ (i.e. such that $\mathcal{A} \ni B \subset A \in \mathcal{U}$ implies $B \in \mathcal{U}$ ). Given a measure $\mu: \mathcal{A} \rightarrow$ $E$, where $E$ is a TVS, the $\mu$-topology is the weakest Fréchet-Nikodym topology on $\mathcal{A}$ which makes $\mu$ (uniformly) continuous. If $U$ ranges over a base of neighborhoods of zero in $E$, then the classes $\mathcal{U}=\{B \in \mathcal{A}$ : $\left.\mu\left(\mathcal{A}_{B}\right) \subset U\right\}$ form a base of solid neighborhoods of $\emptyset$ for the $\mu$-topology. For more information and references, see [D2, Sec. 0].

Proposition 1.5. Let $E$ be a $\sigma$-Dedekind complete TRs. If $\mu: \mathcal{A} \rightarrow E$ is an order bounded measure such that $\mathcal{A}$ is $\mu$-separable, then $|\mu|$ exists properly. Moreover, if $\mathcal{C}$ is a countable $\mu$-dense subalgebra of $\mathcal{A}$, and $\Pi_{\mathcal{C}}(S)$ denotes the countable family of all finite $\mathcal{C}$-partitions of $S$, then

$$
|\mu|(A)=\sup \left\{\sum_{C \in \pi}|\mu(A \cap C)|: \pi \in \Pi_{\mathcal{C}}(S)\right\} \quad \text { for all } A \in \mathcal{A} .
$$

Proof. In view of Proposition 1.4, to verify the first statement it is enough to show that $\sup \mu(\mathcal{A} \cap A)$ exists in $E$ for every $A \in \mathcal{A}$. Let $\mathcal{C}$ be a countable $\mu$-dense subclass of $\mathcal{A}$.

Fix $A \in \Sigma$. Since the map $B \rightarrow A \cap B$ is $\mu$-continuous, $\mathcal{C} \cap A$ is a $\mu$-dense subclass of $\mathcal{A} \cap A$. In consequence, $\mu(\mathcal{C} \cap A)$ is a dense subset of $\mu(\mathcal{A} \cap A)$. Now, the set $\mu(\mathcal{C} \cap A)$, being countable and order bounded, has a supremum, say $z$, in $E$, and it is easily seen that $z$ is also a supremum of $\mu(\mathcal{A} \cap A)$.

We now verify the "moreover" part. Let $\mathcal{C}$ be a countable $\mu$-dense subalgebra of $\mathcal{A}$. Fix $A \in \mathcal{A}$ and denote the supremum in (*) by $z$. Using Proposition 1.2 and applying a continuity-density argument as above, we see that

$$
\begin{aligned}
z & =\sup \left\{\mu\left(A \cap C_{1}\right)-\mu\left(A \cap C_{2}\right): C_{1}, C_{2} \in \mathcal{C}\right\} \\
& =\sup \left\{\mu\left(A \cap B_{1}\right)-\mu\left(A \cap B_{2}\right): B_{1}, B_{2} \in \mathcal{A}\right\}=|\mu|(A),
\end{aligned}
$$

which concludes the proof.

For a $\sigma$-additive measure $\mu$ defined on a $\sigma$-algebra, every generating subalgebra is automatically $\mu$-dense (see [D1, Part III, Thm. 8.2]. Hence as a consequence of the preceding result we have the following. 
Corollary 1.6. Let $E$ be a $\sigma$-Dedekind complete TRS. If $\mu: \Sigma \rightarrow E$ is a $\sigma$-additive order bounded measure and the $\sigma$-algebra $\Sigma$ is countably generated, then the modulus $|\mu|$ exists properly. Moreover, for any countable subalgebra $\mathcal{C}$ generating $\Sigma$, the formula $(*)$ stated above holds for all $A \in \Sigma$.

Evidently, for a bounded measure $\mu: \mathcal{A} \rightarrow \mathbb{R}$ its modulus $|\mu|$ is the same as the (total) variation measure of $\mu$. A reduction to variation measures is also possible when $E$ is a solid sequence space. We first note the following, rather obvious, fact.

Proposition 1.7. Let $E$ be a Riesz subspace of a Riesz space $F$, and let $\mu: \mathcal{A} \rightarrow E$ and $\nu: \mathcal{A} \rightarrow F$ be measures with $\mu(A)=\nu(A)$ for all $A \in \mathcal{A}$.

(a) If $|\nu|$ exists (resp., exists properly) and $|\nu|(\mathcal{A}) \subset E$, then also $|\mu|$ exists (resp., exists properly) and $|\mu|=|\nu|$.

(b) If $E$ is regular in $F$ and $|\mu|$ exists properly, then so does $|\nu|$, and $|\mu|=|\nu|$.

Recall that, in particular, a Riesz subspace $E$ of a Riesz space $F$ is regular if it is order dense in $F$ or is an ideal in $F$.

Corollary 1.8. Let $E$ be a regular Riesz subspace of the Riesz space $\omega$, $\mu=\left(\mu_{n}\right): \mathcal{A} \rightarrow E$ be an order bounded measure, and define a measure $m: \mathcal{A} \rightarrow \omega$ by $m=\left(\left|\mu_{n}\right|\right)$. Then the modulus $|\mu|$ exists properly iff $m(\mathcal{A}) \subset E$, in which case $|\mu|=m$.

In particular, if $E$ is an ideal in $\omega$, then the modulus $|\mu|$ exists properly and $|\mu|=m$.

Proof. Define a measure $\nu: \mathcal{A} \rightarrow \omega$ by $\nu(A)=\mu(A)$. Since $\omega$ is Dedekind complete, $|\nu|$ exists properly and, clearly, $|\nu|=m$. Applying Proposition 1.7 concludes the proof of the main assertion. In order to deduce the other assertion it suffices to note that if $E$ is an ideal and $\mu(\mathcal{A}) \subset[-x, x]$ for some $x \in E_{+}$, then $m(\mathcal{A}) \subset[-2 x, 2 x] \subset E$.

In what follows,

$I=[0,1], \quad(I, \mathcal{B}, \lambda) \quad$ is the standard Borel-Lebesgue measure space, 
$L_{p}=L_{p}(I, \mathcal{B}, \lambda)$ for $1 \leqslant p \leqslant \infty$, and

$\left(r_{n}\right)$ is the Rademacher sequence on $I$.

Recall that $\int_{A} r_{n} d \lambda \rightarrow 0$ for all $A \in \mathcal{B}$.

Example 1.9. In general, the measures that have modulus need not form a vector space. To see this, consider the measures $\mu=\left(\mu_{n}\right), \nu=$ $\left(\nu_{n}\right): \mathcal{B} \rightarrow c$ defined by $\mu_{n}(A)=\int_{A} r_{n} d \lambda$ and $\nu_{n}(A)=(-1)^{n-1} \int_{A} r_{n} d \lambda$. (They are actually $c_{0}$-valued.) Then $\left|\mu_{n}\right|(A)=\left|\nu_{n}\right|(A)=\lambda(A)$ so that, by Corollary 1.8, both $|\mu|$ and $|\nu|$ exist as measures from $\mathcal{B}$ to $c$, and $|\mu|(A)=|\nu|(A)=(\lambda(A), \lambda(A), \ldots)$ for each $A \in \mathcal{B}$. Now consider the measure $\mu+\nu=\left(\mu_{n}+\nu_{n}\right): \mathcal{B} \rightarrow c$. Note that $\mu+\nu=2\left(\mu_{1}, 0, \mu_{3}, 0, \ldots\right)$. Hence if a measure $\xi=\left(\xi_{n}\right): \mathcal{B} \rightarrow c$ is an absolute majorant for $\mu+\nu$, so is the measure $\gamma=\left(\gamma_{n}\right)$, where $\gamma_{n}=\xi_{n}$ when $n$ is odd, and $\gamma_{n}=\left(1-\frac{1}{n}\right) \xi_{n}$ when $n$ is even. Moreover, $\gamma \leqslant \xi$ and $\gamma \neq \xi$ (because $\gamma_{n}(I)<\xi_{n}(I)$ for large even $n$ ). Thus $\mu+\nu$ does not have a modulus.

Note: $\mu+\nu$ is of essentially the same type as the $c$-valued order bounded measure without a modulus given in [hSZ, p. 154].

\section{An order bounded measure without an abso- lute majorant}

In the proposition below, the first part is an immediate consequence of Dunford's characterization of relatively weakly compact subsets of $L_{1}(\mu)$ spaces (see [DU, Thm. III.2.15]). The verification of the second part is straightforward using Corollary 1.8.

Proposition 2.1. The general form of a $\lambda$-continuous measure $\mu$ : $\mathcal{B} \rightarrow \ell_{\infty}$ is $\mu(A)=\left(\int_{A} f_{n} d \lambda\right)$, where $\left(f_{n}\right)$ is a relatively weakly compact sequence in $L_{1}$, and the modulus of such a measure $\mu$ is given by $|\mu|(A)=$ $\left(\int_{A}\left|f_{n}\right| d \lambda\right)$ so that also $|\mu|$ is $\lambda$-continuous.

Remark. Obviously, a measure $\mu$ of the above form is $c_{0}$-valued (resp. $c$-valued) iff the sequence $\left(f_{n}\right)$ is weakly null (resp. weakly convergent).

Lemma 2.2. Let $\left(g_{n}\right)$ be a relatively weakly compact sequence in $L_{1}$. Then there exists a sequence $\left(\rho_{n}\right)$ of $\{-1,1\}$-valued measurable functions on I such that the sequence $\left(f_{n}\right)=\left(g_{n} \rho_{n}\right)$ is weakly null in $L_{1}$.

Proof. As easily seen, without loss of generality we may assume that the functions $g_{n}$ are nonnegative. For every $n$ denote $D_{n}=\{j \in \mathbb{N}: 1 \leqslant j \leqslant$ 
$\left.2^{n}\right\}$ and let $\left\{I_{n j}: j \in D_{n}\right\}$ be a partition of $I$ into subintervals of length $2^{-n}$. For every $n \in \mathbb{N}$ and $j \in D_{n}$ choose $\mathcal{B}$-partitions $I_{n j}=A_{n j} \cup B_{n j}$ so that

$$
\int_{A_{n j}} g_{n} d \lambda=\int_{B_{n j}} g_{n} d \lambda
$$

and define a function $\rho_{n}$ on $I$ by setting $\rho_{n}(t)=-1$ if $t \in \bigcup_{j \in D_{n}} A_{n j}$, and $\rho_{n}(t)=1$ otherwise. Denote $f_{n}=g_{n} \rho_{n}$ and $\varphi_{n}(A)=\int_{A} f_{n} d \lambda$. Clearly, $\varphi_{n}\left(I_{m j}\right)=0$ whenever $n \geqslant m$ and $j \in D_{m}$. In particular,

$$
\lim _{n \rightarrow \infty} \varphi_{n}\left(I_{m j}\right)=0, \quad \text { for all } m \text { and } j \in D_{m} .
$$

Now, by assumption, the sequence $\left(g_{n}\right)$ is relatively weakly compact in $L_{1}$. Equivalently, the indefinite integrals $\int_{(.)} g_{n} d \lambda$ are equi- $\lambda$ continuous. It follows that also the measures $\varphi_{n}$ have the same property. In other words, the functions $\varphi_{n}$ are equicontinuous on the semimetric Fréchet-Nikodym space $\left(\mathcal{B}, d_{\lambda}\right)$, where $d_{\lambda}(A, B)=\lambda(A \Delta B)$. In view of $(*)$ and since the finite unions of the intervals $I_{n j}$ are dense in this space, it follows that $\lim _{n} \varphi_{n}(A)=0$ for every $A \in \mathcal{B}$. Thus the sequence $\left(f_{n}\right)$ is weakly null in $L_{1}$.

Remark. If a sequence $\left(g_{n}\right)$ in $L_{1}$ is relatively (norm) compact, and $\left(r_{n}\right)$ is the usual Rademacher sequence on $I$, then the sequence $\left(f_{n}\right)=\left(g_{n} r_{n}\right)$ is weakly null in $L_{1}$. Indeed, $\left(r_{n}\right) \subset L_{\infty}=L_{1}^{*}$ is weak ${ }^{*}$ null, hence it converges uniformly to zero on every relatively compact subset of $L_{1}$. In particular, $\int_{A} f_{n} d \lambda=\left\langle g_{n} 1_{A}, r_{n}\right\rangle \rightarrow 0$ for every $A \in \mathcal{B}$.

Proposition 2.3. For every positive $\lambda$-continuous measure $\nu: \mathcal{B} \rightarrow \ell_{\infty}$ there exists a $\lambda$-continuous measure $\mu: \mathcal{B} \rightarrow c_{0}$ such that $|\mu|=\nu$.

Proof. By Proposition 2.1, $\nu=\left(\int_{(.)} g_{n} d \lambda\right)$, where $\left(g_{n}\right)$ is a relatively weakly compact sequence in $L_{1}$ consisting of nonnegative functions. Applying Lemma 2.2 , we find a sequence $\left(\rho_{n}\right)$ of $\{-1,1\}$-valued measurable functions on $I$ such that the sequence $\left(f_{n}\right)=\left(g_{n} \rho_{n}\right)$ is weakly null in $L_{1}$. Let $\mu_{n}:=\int_{(.)} f_{n} d \lambda(n \in \mathbb{N})$. Then $\mu:=\left(\mu_{n}\right): \mathcal{B} \rightarrow c_{0}$ is a $\sigma$-additive measure and, by the second part of Proposition 2.1, $|\mu|=\left(\left|\mu_{n}\right|\right)=\nu$.

Example 2.4. There exists a $\lambda$-continuous measure $\mu: \mathcal{B} \rightarrow c_{0} \subset c$ that has no absolute majorant $\gamma: \mathcal{B} \rightarrow c$. (Though, of course, $\mu: \mathcal{B} \rightarrow c$ is order bounded.) 
In view of Proposition 2.3, to prove the existence of such a measure $\mu$, it is enough to find a positive $\lambda$-continuous measure $\nu: \mathcal{B} \rightarrow \ell_{\infty}$ that has no absolute majorant $\gamma: \mathcal{B} \rightarrow c$.

Fix a sequence $1=p_{1}<p_{2}<\ldots$ in $\mathbb{N}$ such that

1) for every $j, p_{j+1}-p_{j}=k^{2}$ for some $k \in \mathbb{N}$;

2) for every $k \in \mathbb{N}$ the set $J_{k}:=\left\{j \in \mathbb{N}: p_{j+1}-p_{j}=k^{2}\right\}$ is infinite.

Note that $\left(J_{k}\right)_{k=1}^{\infty}$ is a partition of $\mathbb{N}$.

For every $k \in \mathbb{N}$ let $\left\{I_{k m}: m=0, \ldots, k^{2}-1\right\}$ be a partition of $I$ into $k^{2}$ subintervals of length $k^{-2}$. Define a sequence of functions $g_{n}: I \rightarrow \mathbb{R}_{+}$ as follows: Given $n$, take the unique $j$ such that $p_{j} \leqslant n<p_{j+1}$. Then $p_{j+1}-p_{j}=k^{2}$ for some $k \in \mathbb{N}$, and $n=p_{j}+m$, where $m \in\left\{0, \ldots, k^{2}-1\right\}$. Set

$$
g_{n}=k \cdot 1_{I_{k m}} .
$$

Clearly, $\left\|g_{n}\right\|_{1}=k^{-1}$, hence the sequence $\left(g_{n}\right)$ is relatively norm compact in $L_{1}$. Define $\nu_{n}=\int_{(\cdot)} g_{n} d \lambda$ for each $n$, and let $\nu:=\left(\nu_{n}\right): \mathcal{B} \rightarrow \ell_{\infty}$. By Proposition 2.1, $\nu$ is a positive $\sigma$-additive measure. Suppose that $\nu$ has a (positive) majorant $\gamma=\left(\gamma_{n}\right): \mathcal{B} \rightarrow c$. Then

$$
\bar{\nu}(A):=\limsup _{n \rightarrow \infty} \nu_{n}(A) \leqslant \lim _{n \rightarrow \infty} \gamma_{n}(A)=: \bar{\gamma}(A) \quad \text { for every } A \in \mathcal{B},
$$

and $\bar{\gamma}: \mathcal{B} \rightarrow \mathbb{R}_{+}$is a measure.

Take any $k \in \mathbb{N}$. If $m=0, \ldots, k^{2}-1$, then $\nu_{p_{j}+m}\left(I_{k m}\right)=k^{-1}$ for every $j \in J_{k}$, hence $\bar{\nu}\left(I_{k m}\right) \geqslant k^{-1}$. Therefore,

$$
k=k^{2} \cdot k^{-1} \leqslant \sum_{m=0}^{k^{2}-1} \bar{\nu}\left(I_{k m}\right) \leqslant \sum_{m=0}^{k^{2}-1} \bar{\gamma}\left(I_{k m}\right)=\bar{\gamma}(I),
$$

or $k \leqslant \bar{\gamma}(I)<\infty$ for every $k \in \mathbb{N}$, which is impossible.

Remarks 2.5. (a) It is worth pointing out that the essence of what we did above was a construction of a relatively norm compact nonnegative sequence $\left(g_{n}\right)$ in $L_{1}$ for which there is no weakly convergent sequence $\left(h_{n}\right)$ in $L_{1}$ such that $g_{n} \leqslant h_{n}$ for all $n$.

(b) Let a measure $\mu: \mathcal{B} \rightarrow c$ be as required in Example 2.4. Consider the induced integral operator $I_{\mu}: L_{\infty}(\lambda) \rightarrow c: I_{\mu}(f)=\int_{I} f d \mu$. It is, of course, order bounded; however, it is not regular. That is, there is 
no positive linear operator $P: L_{\infty}(\lambda) \rightarrow c$ such that $\left|I_{\mu}(f)\right| \leqslant P(f)$ for all $0 \leqslant f \in L_{\infty}(\lambda)$. For, otherwise, the measure $\psi: \mathcal{B} \rightarrow c$ representing $P$ would be an absolute majorant for $\mu$, and $\mu$ does not admit such majorants. See [AB2, Example 1.11] for a different example of an order bounded nonregular operator.

(c) In Example 7.1 below, we construct a $\sigma$-additive order bounded measure on $\mathcal{P}(\mathbb{N})$, with values in a separable Banach lattice, that has no absolute majorant.

In the rest of the paper, unless stated otherwise, we assume that

$$
E=(E, \tau) \text { is a TRS. }
$$

\section{Absolute semivariations and absolute exhaus- tivity}

For a measure $\mu: \mathcal{A} \rightarrow E$ and a Riesz pseudonorm $\rho$ on $E$, we define the absolute semivariation $\mu_{\rho}: \mathcal{A} \rightarrow \overline{\mathbb{R}}_{+}$of $\mu$ relative to $\rho$ by

$$
\mu_{\rho}(A)=\sup _{\pi \in \Pi(A)} \rho\left(\sum_{D \in \pi}|\mu(D)|\right) .
$$

It is easily verified that $\mu_{\rho}$ is a submeasure. In view of Proposition $1.2(\mathrm{~b})$ it is clear that if $\mu$ is order bounded, then $\mu_{\rho}(S)<\infty$ (so $\mu_{\rho}$ assumes only finite values). See also Propositions 3.2 and 3.9 below.

Recall that if $\rho$ is a seminorm, then the (usual) semivariation of $\mu$ relative to $\rho$ is the submeasure $\bar{\mu}_{\rho}: \mathcal{A} \rightarrow \overline{\mathbb{R}}_{+}$that can be defined by any of the equalities below:

$$
\begin{aligned}
\bar{\mu}_{\rho}(A) & =\sup \left\{\rho\left(\sum_{i=1}^{k} \alpha_{i} \mu\left(D_{i}\right)\right):\left\{D_{1}, \ldots, D_{k}\right\} \in \Pi(A),\left|\alpha_{i}\right| \leqslant 1\right\} \\
& =\sup \{\rho(\mu(B)-\mu(C)): B, C \subset A\} .
\end{aligned}
$$

Remembering that for any set $A \in \mathcal{A}$ the net $\left(\sum_{B \in \pi}|\mu(B)|: \pi \in\right.$ $\Pi(A))$ is increasing, and making use of the Fact stated after Proposition 1.1 , one easily verifies the following.

Proposition 3.1. Let $\mu: \mathcal{A} \rightarrow E$ be a measure and $\rho$ a Riesz pseudonorm on $E$. 
(a) If $\gamma$ is an absolute majorant for $\mu$, then $\mu_{\rho}(A) \leqslant \rho(\gamma(A))$ for all $A \in \mathcal{A}$. In particular, if $|\mu|$ exists, then $\mu_{\rho}(A) \leqslant \rho(|\mu|(A))$ for all $A \in \mathcal{A}$.

(b) If $\rho$ has the Fatou property and $|\mu|$ exists properly on a set $A \in \mathcal{A}$, then $\mu_{\rho}(A)=\rho(|\mu|(A))$.

(c) If $\rho$ is a seminorm, then for every $A \in \mathcal{A}, \bar{\mu}_{\rho}(A) \leqslant \mu_{\rho}(A)$; and if $\rho$ is an M-seminorm, then $\bar{\mu}_{\rho}(A)=\mu_{\rho}(A)$.

If $\rho=\|\cdot\|$ is the norm of a Banach lattice $E$, then we shall write $\|\mu\|_{a}(\cdot)$ and $\|\mu\|(\cdot)$ for the associated absolute semivariation and semivariation of $\mu$, respectively.

Remark. In [DW, Thm. 1] it has been shown that for the indefinite Bochner integrals $\mu=\int f d \nu$ in Banach lattices one always has $\|\mu\|_{a}(A)=\left\|\int_{A}|f| d \nu\right\|=\||\mu|(A)\|$.

Proposition 3.2. Let $E=(E,\|\cdot\|)$ be a Banach lattice, $\kappa: E \rightarrow E^{* *}$ the canonical embedding, and $\mu: \mathcal{A} \rightarrow E$ a measure. Then $\|\mu\|_{a}(S)<\infty$ if and only if the measure $\kappa \circ \mu: \mathcal{A} \rightarrow E^{* *}$ is order bounded. Moreover, in this case the modulus $|\kappa \circ \mu|$ exists properly and $\|\mu\|_{a}(A)=\||\kappa \circ \mu|(A)\|$ for every $A \in \mathcal{A}$.

Proof. "If": Since $E^{* *}$ is Dedekind complete and has the Fatou property, $|\kappa \circ \mu|$ exists properly and, by Proposition 3.1 (b), $\|\mu\|_{a}(A)=$ $\|\kappa \circ \mu\|_{a}(A)=\||\kappa \circ \mu|(A)\|$ for all $A \in \mathcal{A}$.

"Only if": Let $A \in \mathcal{A}$. The net $\left(\sum_{D \in \pi}|\mu(D)|: \pi \in \Pi(A)\right)$ is increasing and norm bounded by $\|\mu\|_{a}(A)$. As $E^{* *}$ has the Levi property, $\sup _{\pi} \sum_{D \in \pi}|\mu(D)|$ exists in $E^{* *}$. It follows that $|\kappa \circ \mu|$ exists properly.

Proposition 3.3. If $\mu: \mathcal{A} \rightarrow E$ is a $\sigma$-additive measure and $\rho$ is a continuous Riesz pseudonorm on $E$, then the submeasure $\mu_{\rho}$ is $\sigma$ subadditive.

Proof. Let $\left(A_{n}\right)$ be a disjoint sequence in $\mathcal{A}$ such that also its union $A$ is in $\mathcal{A}$. Denote $B_{N}=\bigcup_{n=N+1}^{\infty} A_{n}$, and let $\pi \in \Pi(A)$. First observe that for each $D \in \pi$ and $N \in \mathbb{N}$ one has $|\mu(D)| \leqslant \sum_{n=1}^{N}\left|\mu\left(A_{n} \cap D\right)\right|+$ 
$\left|\mu\left(B_{N} \cap D\right)\right|$. Hence

$$
\begin{aligned}
\rho\left(\sum_{D \in \pi}|\mu(D)|\right) & \leqslant \sum_{n=1}^{N} \rho\left(\sum_{D \in \pi}\left|\mu\left(A_{n} \cap D\right)\right|\right)+\rho\left(\sum_{D \in \pi}\left|\mu\left(B_{N} \cap D\right)\right|\right) \\
& \leqslant \sum_{n=1}^{\infty} \mu_{\rho}\left(A_{n}\right)+\rho\left(\sum_{D \in \pi}\left|\mu\left(B_{N} \cap D\right)\right|\right) .
\end{aligned}
$$

Since $\mu$ is $\sigma$-additive and $B_{N} \downarrow \emptyset$, the last term on the right tends to zero as $N \rightarrow \infty$, and we easily conclude that $\mu_{\rho}(A) \leqslant \sum_{n=1}^{\infty} \mu_{\rho}\left(A_{n}\right)$.

Let us say that a measure $\mu: \mathcal{A} \rightarrow E$ is absolutely exhaustive if for every disjoint sequence $\left(C_{j}\right)$ in $\mathcal{A}$ the series $\sum_{j}\left|\mu\left(C_{j}\right)\right|$ is Cauchy. Evidently, if $\mu$ admits an exhaustive absolute majorant (in particular, if $|\mu|$ exists and is exhaustive), then $\mu$ is absolutely exhaustive. However, a $\sigma$-additive measure that is absolutely exhaustive need not even be order bounded. Such are, for instance, the $c_{0}$-valued measures in Examples 1.9 and 2.4 .

Proposition 3.4. Let $P$ be a family of Riesz pseudonorms defining the topology of $E$. Then for every measure $\mu: \mathcal{A} \rightarrow E$ the following are equivalent:

(a) For every $\rho \in P$, the absolute semivariation $\mu_{\rho}$ is exhaustive.

(b) $\mu$ is absolutely exhaustive.

Proof. (a) $\Longrightarrow(\mathrm{b})$ : Let $\left(C_{j}\right)$ be a disjoint sequence in $\mathcal{A}$. Since $\mu_{\rho}$ is exhaustive and $\rho\left(\sum_{j=m}^{n}\left|\mu\left(C_{j}\right)\right|\right) \leqslant \mu_{\rho}\left(\bigcup_{j=m}^{n} C_{j}\right)$ for all $n \geqslant m$, the series $\sum_{j}\left|\mu\left(C_{j}\right)\right|$ is Cauchy.

(b) $\Longrightarrow(\mathrm{a})$ : Suppose $\mu_{\rho}$ is not exhaustive. Thus there is a disjoint sequence $\left(A_{n}\right)$ in $\mathcal{A}$ such that $\mu_{\rho}\left(A_{n}\right)>\varepsilon$ for all $n$ and some $\varepsilon>0$. Then, by the definition of $\mu_{\rho}$, for every $n$ there exists $\pi(n) \in \Pi\left(A_{n}\right)$ such that $\rho\left(\sum_{B \in \pi(n)}|\mu(B)|\right)>\varepsilon$. Arrange the families of sets $\pi(1), \pi(2), \ldots$ in a single sequence $C_{1}, C_{2}, \ldots$ so that $\pi(n)=\left\{C_{j}: j \in J(n)\right\}$, where $(J(n))$ is a sequence of consecutive intervals in $\mathbb{N}$. Then $\rho\left(\sum_{j \in J(n)}\left|\mu\left(C_{j}\right)\right|\right)>\varepsilon$ for every $n$. This is impossible because the $C_{j}$ 's are pairwise disjoint and so the series $\sum_{j}\left|\mu\left(C_{j}\right)\right|$ is Cauchy in $E$, by (b).

Proposition 3.5. Let $\mu: \mathcal{A} \rightarrow E$ be a $\sigma$-additive measure and $\rho$ a continuous Riesz pseudonorm on $E$. If the absolute semivariation $\mu_{\rho}$ is exhaustive, then it is also order continuous. 
Proof. This follows from Proposition 3.3 and [D1, Part II, Thm. 5.3]. A direct argument showing this runs as follows: Suppose $\mu_{\rho}$ is not order continuous. Thus there is a sequence $\left(A_{n}\right)$ in $\Sigma$ with $A_{n} \downarrow \emptyset$ such that $\mu_{\rho}\left(A_{n}\right)>\varepsilon$ for all $n$ and some $\varepsilon>0$. Then for every $n$ there exists $\pi(n) \in$ $\Pi\left(A_{n}\right)$ such that $\rho\left(\sum_{B \in \pi(n)}|\mu(B)|\right)>\varepsilon$. Note that $\mu\left(B \backslash A_{n}\right) \rightarrow \mu(B)$ for every $B \in \Sigma$, hence also $\sum_{B \in \pi}\left|\mu\left(B \backslash A_{n}\right)\right| \rightarrow \sum_{B \in \pi}|\mu(B)|$ for every finite $\pi \subset \Sigma$.

Using this, we find by an easy induction a sequence $1=n_{0}<n_{1}<$ $n_{2}<\ldots$ such that

$$
\rho\left(\sum_{B \in \pi\left(n_{k-1}\right)}\left|\mu\left(B \backslash A_{n_{k}}\right)\right|\right)>\varepsilon \quad \text { for } k=1,2, \ldots
$$

Hence $\mu_{\rho}\left(A_{n_{k-1}} \backslash A_{n_{k}}\right)>\varepsilon$ for each $k$, contradicting the exhaustivity of $\mu_{\rho}$.

Corollary 3.6. If a measure $\mu: \mathcal{A} \rightarrow E$ is $\sigma$-additive and admits an exhaustive absolute majorant, then the absolute semivariation $\mu_{\rho}$ is exhaustive and order continuous for every continuous Riesz pseudonorm $\rho$ on $E$.

As a consequence of Propositions 3.4 and 3.5 we also have the following.

Corollary 3.7. Let $P$ be a family of Riesz pseudonorms defining the topology of $E$. Then for a $\sigma$-additive measure $\mu: \Sigma \rightarrow E$ the following are equivalent:

(a) For every $\rho \in P$, the absolute semivariation $\mu_{\rho}$ is order continuous.

(b) For every $\rho \in P$, the absolute semivariation $\mu_{\rho}$ is exhaustive.

(c) $\mu$ is absolutely exhaustive.

Proposition 3.8. If a measure $\mu: \Sigma \rightarrow E$ is $\sigma$-additive and absolutely exhaustive, then $\mu_{\rho}(S)<\infty$ for every continuous Riesz pseudonorm $\rho$ on $E$.

Proof. By Corollary 3.7, $\mu_{\rho}$ is order continuous. Consider a maximal family $\mathcal{C}$ of disjoint sets $C \in \Sigma$ with $0<\mu_{\rho}(C)<\infty$. Clearly, $\mathcal{C}$ has to be countable, say $\mathcal{C}=\left\{C_{1}, C_{2}, \ldots\right\}$. Let $S_{0}=\bigcup \mathcal{C}$. Since $\mu_{\rho}\left(\bigcup_{n \geqslant m} C_{n}\right) \rightarrow$ 0 as $m \rightarrow \infty, \mu_{\rho}\left(S_{0}\right)<\infty$. To finish the proof, it is enough to show that $\mu_{\rho}\left(S \backslash S_{0}\right)=0$. Otherwise $\mu_{\rho}\left(S \backslash S_{0}\right)=\infty$ and, as easily seen, 
$S \backslash S_{0}$ must contain an atom $A$ for $\mu_{\rho}$ with $\mu_{\rho}(A)=\infty$. Then for any $D \subset A$ either $\rho(\mu(D))=0$ or $\rho(\mu(A \backslash D))=0$. It follows that for every $\left\{D_{1}, \ldots, D_{n}\right\} \in \Pi(A)$ we have $\rho\left(\sum_{i}\left|\mu\left(D_{i}\right)\right|\right)=\rho(\mu(A))$. In consequence, $\mu_{\rho}(A)=\rho(\mu(A))<\infty$; a contradiction.

Applying Proposition 3.8 and Corollary 3.7 in the setting of Proposition 3.2 , we have the following complement to the latter result.

Corollary 3.9. Let $E$ be a Banach lattice, and $\kappa: E \rightarrow E^{* *}$ the canonical embedding. If a measure $\mu: \Sigma \rightarrow E$ is $\sigma$-additive and absolutely exhaustive, then the measure $\kappa \circ \mu: \Sigma \rightarrow E^{* *}$ has a $\sigma$-additive proper modulus $|\kappa \circ \mu|$.

\section{Topological Riesz spaces with Properties (S) and $(\mathrm{CS})$}

Recall that $E=(E, \tau)$ is a TRS. Consider a series $(*) \sum_{n=1}^{\infty} x_{n}$ in $E$.

We shall say that the series $(*)$ is perfectly order bounded if the set of all its finite sums, $\left\{\sum_{n \in M} x_{n}: M \subset \mathbb{N}\right.$ finite $\}$, is order bounded. In view of the fact stated before Proposition 1.2, this is equivalent to the requirement that the series $\sum_{n}\left|x_{n}\right|$ is order bounded, that is, the sequence $\sum_{i=1}^{n}\left|x_{i}\right|(n \in \mathbb{N})$ of its partial sums is order bounded.

Now assume that the series $(*)$ is subseries convergent in $E$. Then we define the associated ( $\sigma$-additive) measure $\xi: \mathcal{P}(\mathbb{N}) \rightarrow E$ by $\xi(M)=$ $\sum_{n \in M} x_{n}$. As easily seen, the series $(*)$ is perfectly order bounded iff the measure $\xi$ is order bounded.

The following extends a result from [DW, Cor.], where $E$ was a Banach lattice. It can be easily verified by a direct argument, or deduced from Propositions 6.4 and 8.6 below.

Proposition 4.1. Let $\sum_{n} x_{n}$ be a subseries convergent series in $E$ with the associated measure $\xi: \mathcal{P}(\mathbb{N}) \rightarrow E$. Then the following hold.

(a) The modulus $|\xi|$ exists properly iff the series $\sum_{n}\left|x_{n}\right|$ is subseries (o)-convergent, in which case

$$
|\xi|(M)=(\mathrm{o})-\sum_{n \in M}\left|x_{n}\right| \quad \text { for all } M \subset \mathbb{N} ;
$$

(b) The modulus $|\xi|$ exists and is $\sigma$-additive iff the series $\sum_{n}\left|x_{n}\right|$ is 
subseries convergent, in which case

$$
|\xi|(M)=\sum_{n \in M}\left|x_{n}\right| \quad \text { for all } M \subset \mathbb{N},
$$

and hence $|\xi|$ exists properly.

We now introduce a few definitions.

We shall say that the TRS $E$ (or its topology) has Property (S) if for every subseries convergent and perfectly order bounded series $\sum_{n} x_{n}$ in $E$, the series $\sum_{n}\left|x_{n}\right|$ is convergent. Note that then every subseries of the latter series must be convergent. In other words, $E$ has Property (S) iff for every order bounded $\sigma$-additive measure $\mu: \mathcal{P}(\mathbb{N}) \rightarrow E$ its modulus $|\mu|$ exists and is $\sigma$-additive. As was already mentioned in the Introduction, for Banach lattices $E$ this property was introduced by Groenewegen $[\mathrm{G}]$ as property $(\mathrm{C})$; see also [GvR], where such $E$ are said to be order bounded $A M$. Some characterizations of this property, mostly of measure or operator theoretic type, were given in [G, Ch. III and $\mathrm{V}]$ and $[\mathrm{GvR}]$, and more recently in [CW, Thm. 2.6].

A Cauchy type version of $(\mathrm{S})$ will also be useful. But first recall that a series $\sum_{n} x_{n}$ is called unconditionally Cauchy if for every neighborhood $U$ of zero there is a finite set $M \subset \mathbb{N}$ such that $\sum_{n \in N} x_{n} \in U$ for all finite sets $N \subset \mathbb{N}$ disjoint from $M$. This is equivalent to requiring that every subseries $\sum_{k} x_{n_{k}}$ of the series $\sum_{n} x_{n}$ is Cauchy (in the usual sense). Obviously, for positive series "unconditionally Cauchy" and "Cauchy" is the same. Now, we shall say that the TRS E (or its topology) has Property (CS) if for every unconditionally Cauchy and perfectly order bounded series $\sum_{n} x_{n}$ in $E$, the series $\sum_{n}\left|x_{n}\right|$ is (unconditionally) Cauchy. Evidently, if $E$ is sequentially complete, or merely has sequentially complete order intervals (comp. with [AB1, Thms. 13.2 and 17.6]), then Properties (S) and (CS) are equivalent.

We will also need a weaker, "order bounded" variant of the $(\sigma-)$ monotone completeness property, $(\sigma-)$ MCP (see [AB1, Def. 7.4 and Thm. 16.1]). We shall say the TRS $E$ has the $\sigma$-MCP for intervals, if every monotone order bounded Cauchy sequence in $E$ is convergent; equivalently, every Cauchy and order bounded positive series in $E$ is convergent. The general MCP for intervals is defined similarly, using nets in place of sequences. 
This type of completeness goes back to Veksler's paper [V], and was later studied in [La]. It is known (see [V] and [La, Prop. 5.2]) that a metrizable Riesz space $E$ is intervally complete, i.e., has (topologically) complete order intervals, iff $E$ has the $\sigma$-MCP for intervals. In consequence, if a TRS $E$ has the $\sigma$-MCP for intervals, then it is relatively uniformly complete. That is, for every $0 \leqslant u \in E$ the ideal $E_{u}$ generated by $u$ is a Banach space under the norm $\|\cdot\|_{u}$ whose closed unit ball is equal to the order interval $[-u, u]$.

Proposition 4.2. (a) If $E$ has Property (CS), then every exhaustive order bounded measure $\mu: \mathcal{A} \rightarrow E$ is absolutely exhaustive.

(b) If $E$ has the $\sigma-M C P$ for intervals, $\Sigma$ is an infinite $\sigma$-algebra, and every $\sigma$-additive order bounded measure $\mu: \Sigma \rightarrow E$ is absolutely exhaustive, then E has Property (S).

Proof. (b): Let $\sum_{n} x_{n}$ be a subseries convergent and perfectly order bounded series in $E$. Since $\Sigma$ is infinite, we may choose a disjoint sequence $\left(A_{n}\right)$ in $\Sigma \backslash\{\emptyset\}$, and for each $n$ pick a point $s_{n} \in A_{n}$. Define $\mu: \Sigma \rightarrow E$ by $\mu(A)=\sum_{n} 1_{A}\left(s_{n}\right) x_{n}$. Then $\mu$ is a $\sigma$-additive and order bounded measure. By assumption, $\mu$ is absolutely exhaustive. Hence, the series $\sum_{n}\left|\mu\left(A_{n}\right)\right| \equiv \sum_{n}\left|x_{n}\right|$ is Cauchy in $E$. Since it is also order bounded, and $E$ has the $\sigma$-MCP for intervals, it is convergent.

Proposition 4.3. Assume that $E$ has both the $\sigma-M C P$ for intervals and Property (CS). Then for every unconditionally Cauchy and perfectly order bounded series $\sum_{n} x_{n}$ in $E$, both the series $\sum_{n} x_{n}$ and $\sum_{n}\left|x_{n}\right|$ are subseries convergent. In particular, E has Property (S).

Proof. In view of (CS), every subseries of the series $\sum_{n}\left|x_{n}\right|$ is Cauchy and order bounded, hence converges in $E$, by the $\sigma$-MCP for intervals. Since $\sum_{i=m}^{n} x_{i}^{ \pm} \leqslant \sum_{i=m}^{n}\left|x_{i}\right|$ whenever $m \leqslant n$, the positive series $\sum_{n} x_{n}^{+}$ and $\sum_{n} x_{n}^{-}$are Cauchy and order bounded. Applying again the $\sigma$-MCP for intervals, it follows that the series $\sum_{n} x_{n}$ is subseries convergent.

Corollary 4.4. Assume that $E$ has the $\sigma-M C P$ for intervals and Property (CS), and let $\mu: \mathcal{A} \rightarrow E$ be an exhaustive order bounded measure. Then for every disjoint sequence $\left(D_{n}\right)$ in $\mathcal{A}$ both the series $\sum_{n} \mu\left(D_{n}\right)$ and $\sum_{n}\left|\mu\left(D_{n}\right)\right|$ are convergent, and for every monotone sequence $\left(A_{n}\right)$ in $\mathcal{A}$ the limit $\lim _{n} \mu\left(A_{n}\right)$ exists.

As will be seen from Propositions 4.5, 4.7 and 4.8, the classes of 
TRS's with Property (S) or (CS) are fairly wide. In particular, every AM-space has Property (S), because:

Proposition 4.5. For a Banach lattice $E=(E,\|\cdot\|)$ the following are equivalent:

(a) E is Riesz homeomorphic to an AM-space.

(b) For every unconditionally convergent series $\sum_{n} x_{n}$ in $E$, the series $\sum_{n}\left|x_{n}\right|$ is convergent.

(c) Every unconditionally convergent series in $E$ is order bounded.

(d) For every unconditionally convergent series $\sum_{n} x_{n}$ in $E$, the series $\sum_{n}\left|x_{n}\right|$ is norm bounded (i.e., the sequence of its partial sums is norm bounded).

Proof. (a) $\Longrightarrow$ (b): We may assume that $E$ is an AM-space. Let $\sum_{n} x_{n}$ be an unconditionally convergent series in $E$. Since the norm in $E$ is an $M$-norm, using the fact stated before Proposition 1.2 (or, alternatively, Proposition 3.1 (c)) we see that

$$
\left\|\sum_{n \in J}\left|x_{n}\right|\right\| \leqslant 2 \sup _{K \subset J}\left\|\sum_{n \in K} x_{n}\right\| \quad \text { for every finite subset } J \text { of } \mathbb{N} .
$$

It follows that the series $\sum_{n}\left|x_{n}\right|$ converges.

(b) $\Longrightarrow(\mathrm{c})$ and $(\mathrm{c}) \Longrightarrow(\mathrm{d})$ are obvious.

$(\mathrm{d}) \Longrightarrow(\mathrm{a})$ : By Theorem 3.2 in [APY], $E$ is isomorphic to an AMspace (or admits an equivalent M-norm) if and only if the following holds:

(*) There exists a constant $d>0$ such that

$$
\left\|\sum_{i=1}^{n}\left|x_{i}\right|\right\| \leqslant d \sup \left\{\left\|\sum_{i=1}^{n} \varepsilon_{i} x_{i}\right\|: \varepsilon_{i}= \pm 1\right\} \quad \text { for all } x_{1}, \ldots, x_{n} \text { in } E .
$$

Suppose $(*)$ is false. Then we can find an infinite sequence $\left(x_{n}\right)$ in $E$ such that for a certain partition $\left(J_{k}\right)$ of $\mathbb{N}$ into consecutive intervals,

$\sup \left\{\left\|\sum_{i \in J_{k}} \varepsilon_{i} x_{i}\right\|: \varepsilon_{i}= \pm 1\right\} \leqslant 2^{-k}$ and $\left\|\sum_{i \in J_{k}}\left|x_{i}\right|\right\|=k, \quad k=1,2, \ldots$ 
Clearly, the series $\sum_{n} x_{n}$ is unconditionally convergent, while the series $\sum_{n}\left|x_{n}\right|$ is not norm bounded. A contradiction.

Remark. The implication (a) $\Longrightarrow$ (b) is a result of Jameson [Ja2, Prop. 5], while (b) $\Longrightarrow$ (a) was shown in [APY, Thm. 3.2]. See also [CL, Cor. 2], [Le, Cor. 2.2], and [hS, IV, ex. 16]. The equivalence of (a) and (c) was established in [G, Thm. III.4.8]. It is worth pointing out that Jameson's result is essentially an abstract version of an old result due to W. Sierpiński (1910 for real functions; [S] for complex functions): A series of functions $\sum_{n} f_{n}$ is unconditionally uniformly convergent iff the series $\sum_{n}\left|f_{n}\right|$ is uniformly convergent. Similar results for the subseries a.e. uniform convergence of series of bounded measurable or continuous functions were obtained by Orlicz [O; Thms. 5, 6, and 7].

We note the following consequence of Proposition 4.5.

Corollary 4.6. For a Banach lattice $E$ the following are equivalent:

(a) E is Riesz homeomorphic to an AM-space.

(b) Every $\sigma$-additive measure $\mu: \mathcal{P}(\mathbb{N}) \rightarrow E$ is absolutely exhaustive.

(c) Every $\sigma$-additive E-valued measure defined on a $\sigma$-algebra is absolutely exhaustive.

By an M-topological Riesz space (M-TRS) we shall mean a TRS E whose topology can be defined by a family of Riesz M-seminorms or, equivalently, a TRS $E$ which is Riesz homeomorphic to a Riesz subspace of the product of a family of AM-spaces. (Cf. [Ja1] and [C, Cor. 5.8.24].)

We shall say that a Riesz subspace $F$ of a TRS $E$ is sequentially obmclosed in $E$ if, whenever a monotone (or just increasing) sequence in $F$ is order bounded in $F$ and converges in $E$, then its limit is actually in $F$. Equivalently, whenever a positive series in $F$ is order bounded in $F$ and converges in $E$, its sum is in $F$. Evidently, if $F$ is an ideal in $E$, or $F$ is sequentially closed in $E$, or $F$ is sequentially order closed in $E$, then $F$ is sequentially obm-closed in $E$. Note that, in the space of bounded real functions on $[0,1]$ considered with the topology of pointwise convergence, the subspace consisting of Borel measurable functions is both sequentially closed and sequentially order closed, but not an ideal. Furthermore, the linear span of the unit vectors in $\ell_{\infty}$ is a Riesz subspace that is neither topologically nor order sequentially closed. However, it is 
sequentially obm-closed; in fact, its order intervals are even norm complete. This example provides also a simple illustration to part (a) of the proposition below.

Proposition 4.7. The following statements hold.

(a) If a TRS has Property (S), so do all its sequentially obm-closed Riesz subspaces.

(b) The product of any family of TRS with Property (S) has Property $(\mathrm{S})$.

(c) Every M-TRS with the $\sigma$-MCP for intervals has Property (S).

(d) If a TRS is $\sigma$-Dedekind complete and $\sigma$-Lebesgue, then it has the $\sigma-M C P$ for intervals and Property (S).

Exact analogues of statements (a), (b) and (c) are also valid for Property (CS), with "sequentially obm-closed" in (a), and "the $\sigma$-MCP for intervals" in (c) omitted. In addition,

(e) If a TRS is pre-Lebesgue (in particular, Lebesgue), then it has Property (CS).

Proof. Statements (a) and (b) are obvious, and (c) follows easily from Proposition 4.5. As for (d), note that a TRS $E$ is $\sigma$-Dedekind complete and $\sigma$-Lebesgue iff every order bounded positive series in $E$ is convergent. Finally, if $E$ is Lebesgue, then it is pre-Lebesgue (see [AB1, Thm. 10.2]), and the latter property implies (CS) in a trivial way.

The following two easy facts are also worth mentioning.

\section{Proposition 4.8.}

(a) If $\left\{\tau_{\alpha}: \alpha \in A\right\}$ is a family of locally solid topologies on a Riesz space $E$, each having Property (CS), then also the supremum topology, $\sup _{\alpha} \tau_{\alpha}$, has Property (CS).

(b) Let $\left(E_{\alpha}, \tau_{\alpha}\right), \alpha \in A$, be a family of TRS's having Property (S) and such that each $\left(E_{\alpha}, \tau_{\alpha}\right)$ is continuously included as a Riesz subspace in a Riesz space $F$ equipped with a Hausdorff topology $\tau_{F}$. Then the TRS $(E, \tau)$, where $E=\bigcap_{\alpha} E_{\alpha}$ and $\tau=\sup _{\alpha}\left(\tau_{\alpha} \mid E\right)$, has Property (S). 


\section{The $\sigma$-MCP for intervals and positive copies of $c$ or $\ell_{\infty}$}

We first prove a result that is complementary to analogous characterizations obtained in [DL, Sec. 2].

Proposition 5.1. For a TRS $E$ the following are equivalent.

(a) $E$ is $\sigma$-Dedekind complete and $\sigma$-Lebesgue.

(b) E has the $\sigma-M C P$ for intervals and contains no positive copy of $c$; that is, there is no positive linear isomorphic embedding $T: c \rightarrow E$.

Proof. (b) $\Longrightarrow(a)$ : Suppose (a) is false. Then (see the proof of Proposition 4.7), there is a nonconvergent positive series $\sum_{n} x_{n}$ in $E$ such that $x_{1}+\cdots+x_{n} \leqslant x$ for some $x \in E$ and all $n$. Then the series is not Cauchy, because $E$ has the $\sigma$-MCP for intervals. Hence we may assume without loss of generality that $x_{n} \nrightarrow 0$. Let $a=\left(\alpha_{n}\right) \in c_{0}$. Then

$$
\sum_{i=m}^{n}\left|\alpha_{i} x_{i}\right| \leqslant \sup _{m \leqslant i \leqslant n}\left|\alpha_{i}\right| \sum_{i=m}^{n}\left|x_{i}\right| \leqslant \sup _{m \leqslant i \leqslant n}\left|\alpha_{i}\right| \cdot x \quad \text { whenever } m \leqslant n,
$$

hence the positive series $\sum_{n}\left|\alpha_{n} x_{n}\right|$ is Cauchy. As in the proof of Proposition 4.3 , it follows that the series $\sum_{n} \alpha_{n} x_{n}$ converges in $E$. Clearly, the formula $T_{0}(a)=\sum_{n} \alpha_{n} x_{n}$ defines a positive linear operator from $c_{0}$ to $E$ mapping the unit ball of $c_{0}$ into the interval $[-x, x]$. Hence $T_{0}$ is also continuous, and $T_{0}\left(e_{n}\right)=x_{n} \nrightarrow 0$. By [D3], there exists an infinite subset $M$ of $\mathbb{N}$ such that $T_{0}$ is an isomorphic (and positive) embedding of $c_{0}(M) \cong c_{0}$ into $E$.

We may assume of course that $M=\mathbb{N}$ and that $x \notin T_{0}\left(c_{0}\right)$. Define a linear operator $T: c \rightarrow E$ as follows: Given $a=\left(\alpha_{n}\right) \in c$, denote $\alpha_{\infty}=\lim _{n} \alpha_{n}$ and $a_{0}=\left(\alpha_{n}-\alpha_{\infty}\right) \in c_{0}$, and then set

$$
T(a)=\alpha_{\infty} x+T_{0}\left(a_{0}\right)=\alpha_{\infty} x+\sum_{n=1}^{\infty}\left(\alpha_{n}-\alpha_{\infty}\right) x_{n} .
$$

If $a \geqslant 0$, then for every $n$,

$$
\alpha_{\infty} x+\sum_{i=1}^{n}\left(\alpha_{i}-\alpha_{\infty}\right) x_{i}=\alpha_{\infty}\left(x-\sum_{i=1}^{n} x_{i}\right)+\sum_{i=1}^{n} \alpha_{i} x_{i} \geqslant 0
$$


and it follows that $T(a) \geqslant 0$. Thus $T$ is positive. Let $e_{\infty}=(1,1,1, \ldots) \in$ $c$. Then $T$ maps isomorphically each summand in $c=\mathbb{R} \cdot e_{\infty} \oplus c_{0}$ onto the corresponding summand in $T(c)=\mathbb{R} \cdot x \oplus T_{0}\left(c_{0}\right)$, hence $T$ is an isomorphic embedding.

(a) $\Longrightarrow(\mathrm{b})$ : That $E$ has to enjoy the $\sigma$-MCP for intervals is obvious. Suppose $T: c \rightarrow E$ is a positive isomorphic embedding, and denote $x_{n}=$ $T e_{n}$. Then $x_{1}+\cdots+x_{n} \leqslant T\left(e_{\infty}\right)$ for each $n$ so that the positive series $\sum_{n} x_{n}$ is order bounded. However, it is not convergent because $x_{n} \nrightarrow 0$, which contradicts (a).

Remark 5.2. For $E$ a Banach lattice the above result states that $E$ is Lebesgue (or has order continuous norm) iff $E$ contains no positive copy of c (comp. [AB2, Thms. 14.9, 14.21]). It is worth noting that " $E$ contains no positive copy of $c$ " cannot be replaced here with " $E$ contains no Riesz homeomorphic copy of $c$ ". Indeed, the Banach lattice $C([0,1])$ is not Lebesgue, and though it obviously contains a positive copy of $c$, it contains no Riesz homeomorphic copy of c.

For, suppose it does. Then there are a disjoint sequence $\left(f_{n}\right)$ in $C[0,1]$ and an $f \in C[0,1]$ such that $\left\|f_{n}\right\|>1,0 \leqslant f_{n} \leqslant f$, and $(f-$ $\left.f_{n}\right) \wedge f_{n}=0$ for all $n$. For each $n$ choose a point $t_{n} \in[0,1]$ so that $f_{n}\left(t_{n}\right)=\left\|f_{n}\right\|$, and let $\left[a_{n}, b_{n}\right]$ be a maximal closed interval containing $t_{n}$ and such that $f(t)>0$ for $t \in\left(a_{n}, b_{n}\right)$. Observe that $f\left(s_{n}\right)=0$ for some $s_{n} \in\left\{a_{n}, b_{n}\right\}$ and that $b_{n}-a_{n} \rightarrow 0$ as $n \rightarrow \infty$. In consequence, also $t_{n}-s_{n} \rightarrow 0$. Now, $f\left(t_{n}\right)-f\left(s_{n}\right)=f_{n}\left(t_{n}\right)-f_{n}\left(s_{n}\right)=f_{n}\left(t_{n}\right)>1$ for every $n$, contradicting the uniform continuity of $f$.

The next result is taken from [DL, Prop. 2.8]; it will be of decisive importance in the proof of Theorem 6.7 in the next section.

Proposition 5.3. Let a TRS $E$ be relatively uniformly complete (in particular, let $E$ have the $\sigma-M C P$ for intervals). Then $E$ contains no positive copy of $\ell_{\infty}$ iff every measure $\nu: \mathcal{P}(\mathbb{N}) \rightarrow E_{+}$is exhaustive.

\section{$6 \quad$ Exhaustivity and $\sigma$-additivity of the modulus of a measure}

We first tackle the problem of exhaustivity and $\sigma$-additivity of the modulus by applying the results of the preceding section, thus implicitly using the associated absolute semivariations $\mu_{\rho}$. Theorem 6.2 (i) (b) and 
Theorem 6.2 (ii) (b) below are generalizations of the results first proved by Schaefer and Zhang in [hSZ, Thm. 5] in the case of Banach lattices, with the latter result for the particular case of Dedekind complete AMspaces with units. For the same particular case, Theorem 6.2 (ii) (a) was obtained earlier by Schmidt [kS2, Thm. 1.3].

Directly from Propositions 3.1 (b) and 3.4, and Corollary 3.7 we have the following.

Proposition 6.1. Let E have the Fatou property.

(a) If a measure $\mu: \mathcal{A} \rightarrow E$ has a proper modulus $|\mu|$, then $|\mu|$ is exhaustive iff $\mu$ is absolutely exhaustive.

(b) If a $\sigma$-additive measure $\mu: \Sigma \rightarrow E$ has a proper modulus $|\mu|$, then $|\mu|$ is $\sigma$-additive iff $\mu$ is absolutely exhaustive.

Combining the above with Propositions 4.2 and 4.7 we obtain the following result. (Recall that if a TRS is Lebesgue, then it is also Fatou, see [AB1, Thm. 11.6].)

Theorem 6.2. If E has both the Fatou property and Property (CS), in particular,

(i) E is Lebesgue, or

(ii) $E$ is an $M$-TRS with the Fatou property,

then the following statements hold.

(a) If an exhaustive measure $\mu: \mathcal{A} \rightarrow E$ has a proper modulus $|\mu|$, then $|\mu|$ is exhaustive.

(b) If a $\sigma$-additive measure $\mu: \Sigma \rightarrow E$ has a proper modulus $|\mu|$, then $|\mu|$ is $\sigma$-additive.

Remark. In the case (i) statement (a) could be formulated in a stronger form: If a measure $\mu: \mathcal{A} \rightarrow E$ has a proper modulus $|\mu|$, then $|\mu|$ is exhaustive. In fact, by the Lebesgue property, every positive order bounded measure with values in $E$ is automatically exhaustive. 
In the remaining part of this section we deal with the above problem using more direct methods. Our first result, Theorem 6.3, is a variant of Theorem 6.2 (ii). In proving the proper existence of $|\mu|$, we follow closely the ideas already used in $[\mathrm{kS} 1, \mathrm{Thm} .5 .3]$ (and later in [DW, Ex. 1, (b)]) for $E$ an AM-space. Theorem 6.3, (b) for $E$ an AM-space, with $\mu$ assumed additionally to be order bounded, was obtained in [hSZ, Cor. on p. 157] (cf. also [kS3, Thm. 5.2.7]).

Theorem 6.3. Let $E$ be a quasi-complete M-TRs.

(a) If a measure $\mu: \mathcal{A} \rightarrow E$ has relatively compact range, then $|\mu|$ exists properly and is exhaustive.

(b) If a measure $\mu: \Sigma \rightarrow E$ has relatively compact range and is $\sigma$ additive, then $|\mu|$ exists properly and is $\sigma$-additive.

Proof. (b): For $A \in \Sigma$ denote by $\mathfrak{F}_{A}$ the class of all finite families $\Delta$ of sets from $\Sigma_{A}$ directed upward by inclusion. Furthermore, let $M_{A}=$ $\left\{\sup _{B, C \in \Delta}|\mu(B)-\mu(C)|: \Delta \in \mathfrak{F}_{A}\right\}$.

Now, by assumption, $E$ is a quasi-complete M-TRS and the range of $\mu$ is relatively compact. Therefore, by [AB2, Thm. 12.29], which can be generalized easily to quasi-complete M-TRS's, also the set $M_{A}$ is relatively compact, hence its sup exists and belongs to the closure of $M_{A}$. Evidently, $\sup M_{A}=\sup \{|\mu(B)-\mu(C)|: B, C \subset A\}$. It follows that $|\mu|$ exists properly and that $|\mu|(A)=\sup M_{A}$ for all $A \in \Sigma$. Moreover, by compactness, $|\mu|(A)=(\tau)-\lim _{\Delta} \sup _{B, C \in \Delta}|\mu(B)-\mu(C)|$. In consequence, for every continuous M-seminorm $\rho$ on $E$,

$$
\rho(|\mu|(A)) \leqslant \sup _{\Delta} \sup _{B, C \in \Delta} \rho(\mu(B)-\mu(C)) \leqslant 2 \sup _{B \subset A} \rho(\mu(B)) .
$$

From this the $\sigma$-additivity of $|\mu|$ follows immediately.

The proof of (a) is similar noting that the relative compactness of $\mu(\mathcal{A})$ implies $\mu$ is exhaustive (by a result in $[\mathrm{R}]$ ).

Remark. In the theorem above $E$ is assumed quasi-complete. However, it is not hard to see that, in general, for a TRS the notions of completeness and quasi-completeness coincide (see [We, Prop. 2.8], [W2, Prop. 1.4]).

Proposition 6.4. Let $\mu: \mathcal{A} \rightarrow E$ be a $\sigma$-additive measure such that the modulus $|\mu|$ exists. Assume that whenever $A_{n} \uparrow A$ in $\mathcal{A}$, the limit $\lim _{n}|\mu|\left(A_{n}\right)$ exists in $E$. Then $|\mu|$ is $\sigma$-additive. 
Proof. Let $A_{n} \uparrow A$ in $\mathcal{A}$. Define a measure $\nu: \mathcal{A}_{A} \rightarrow E$ by $\nu(B)=$ $\lim _{n}|\mu|\left(A_{n} \cap B\right)$. Obviously, $\nu \leqslant|\mu| \mid \mathcal{A}_{A}$. Since $|\mu(B)|=\lim _{n} \mid \mu\left(A_{n} \cap\right.$ $B) \mid \leqslant \nu(B)$ for each $B \in \mathcal{A}_{A}$, the measure $\nu$ is an absolute majorant for $\mu \mid \mathcal{A}_{A}$. From this and Proposition 1.1 it follows that $|\mu| \mid \mathcal{A}_{A} \leqslant \nu$ and, consequently, $\nu=|\mu| \mid \mathcal{A}_{A}$. In particular, $\nu(A)=|\mu|(A)$.

Note that the condition imposed on $|\mu|$ above is satisfied when $|\mu|$ is exhaustive and $E$ has the $\sigma$-MCP for intervals. It is so in particular when $E$ is $\sigma$-Dedekind complete and $\sigma$-Lebesgue. In fact, in the latter case, it follows from Corollary 1.3 that every order bounded measure $\mu: \mathcal{A} \rightarrow E$ is exhaustive. Hence Proposition 6.4 has the following two consequences of which the second is a remarkable improvement of Theorem 6.2 (i).

Corollary 6.5. Let $E$ have the $\sigma-M C P$ for intervals. If $\mu: \mathcal{A} \rightarrow E a$ $\sigma$-additive measure such that the modulus $|\mu|$ exists and is exhaustive, then it is also $\sigma$-additive.

Corollary 6.6. Let $E$ be $\sigma$-Lebesgue and $\sigma$-Dedekind complete. If $\mu: \mathcal{A} \rightarrow E$ is a $\sigma$-additive measure such that the modulus $|\mu|$ exists, then $|\mu|$ is $\sigma$-additive.

Our next result is also an improvement of Theorem 6.2 (i) and, at the same time, a generalization of a very special case of a result due to Kronsbein and Meyer-Nieberg [KM-N, Thm. 11] for $E$ a Banach lattice without isomorphic copies of $\ell_{\infty}$. (In that case a theorem of Diestel and Faires [DU, Thm. I.4.2] can be used in place of our Proposition 5.3.)

Theorem 6.7. Let $E$ have the $\sigma-M C P$ for intervals and contain no positive copy of $\ell_{\infty}$. If $\mu: \Sigma \rightarrow E$ is a $\sigma$-additive measure such that the modulus $|\mu|$ exists, then $|\mu|$ is $\sigma$-additive.

Proof. By Proposition 5.3, $|\mu|$ is exhaustive. To finish, apply Corollary 6.5.

Remark. The noncontainment of a positive copy of $\ell_{\infty}$ is by no means necessary for the assertion of Theorem 6.7 to hold. This should be clear from Theorem 6.2 (ii) (or [hSZ, Thm. 5(1)]). In fact, it is rather straightforward to see that for every $\sigma$-additive measure $\mu=\left(\mu_{n}\right): \Sigma \rightarrow$ $\ell_{\infty}$ its modulus $|\mu|=\left(\left|\mu_{n}\right|\right)$ is also $\sigma$-additive. (Uniform $\sigma$-additivity or exhaustivity of the $\mu_{n}$ 's implies the same property for the $\left|\mu_{n}\right|$ 's.)

We conclude with a result that can be regarded as a complement to Corollary 6.5. 
Proposition 6.8. Let $E$ have the $M C P$ for intervals. If a $\sigma$-additive measure $\mu: \mathcal{A} \rightarrow E$ admits an exhaustive absolute majorant $\gamma$, then it also admits a smaller $\sigma$-additive absolute majorant $\nu$.

Proof. Let $\left(D_{n}\right)$ be a disjoint sequence in $\mathcal{A}$ with union $A \in \mathcal{A}$. Then $\left|\mu\left(D_{1} \cup \cdots \cup D_{k}\right)\right| \leqslant \gamma\left(D_{1}\right)+\cdots+\gamma\left(D_{k}\right) \leqslant \gamma(A)$. Hence, since $\mu$ is $\sigma$-additive,

$$
|\mu(A)| \leqslant \sum_{n=1}^{\infty} \gamma\left(D_{n}\right) \leqslant \gamma(A),
$$

where the series is $\tau$-convergent.

Let $\nu$ be the $\sigma$-additive summand in the Hewitt-Yosida decomposition of $\gamma$. Recall that, for every $A \in \mathcal{A}$,

$$
\nu(A)=\tau-\lim _{\pi} \sum_{D \in \pi} \gamma(D)
$$

where the limit is taken over the net of all countable $\mathcal{A}$-partitions $\pi$ of $A$. (See [D2, Sec. 3]; in the present setting, the MCP for intervals in $E$ ensures the existence of the limit above.) From $(*)$ it follows that $|\mu(A)| \leqslant \nu(A) \leqslant \gamma(A)$, which concludes the proof.

\section{$7 \quad \sigma$-additive measures with a non- $\sigma$-additive mod- ulus}

At the end of their paper [hSZ], Schaefer and Zhang raised the problem of whether the modulus of a $\sigma$-additive order bounded measure $\mu$ with values in a Dedekind complete Banach lattice is always $\sigma$-additive. In [DW, Ex. 3], we showed that it is not so for the Banach lattice $\ell_{\infty}\left(L_{2}\right)=\left(L_{2} \oplus L_{2} \oplus \ldots\right)_{\ell_{\infty}}$, where $L_{2}=L_{2}[0,1]$, even if the domain of $\mu$ is the $\sigma$-algebra $\mathcal{P}(\mathbb{N})$. That is, we proved that the Dedekind complete Banach lattice $\ell_{\infty}\left(L_{2}\right)$ lacks Property $(S)$ by exhibiting an unconditionally convergent and perfectly order bounded series $\sum_{n} f_{n}$ in $\ell_{\infty}\left(L_{2}\right)$ such that the series $\sum_{n}\left|f_{n}\right|$ is not convergent. In our construction, the terms of the "bad" series $\sum_{n} f_{n}$ come up in consecutive blocks $\left(f_{n}: n \in J_{k}\right)$, where the $k$ th block is formed by the first $n_{k}$ Rademacher functions in the $k$ th copy of $L_{2}$, each multiplied by $n_{k}^{-1}$, and $n_{k} \rightarrow \infty$. Since, for each $k$, we have $\left\|\sum_{n \in J} f_{n}\right\|_{2} \leqslant n_{k}^{-1 / 2}$ for $J \subset J_{k}$, while $\sum_{n \in J_{k}}\left|f_{n}\right|=1=1_{[0,1]}$, the series $\sum_{n} f_{n}$ is as required. 
We take this opportunity to acknowledge the fact that the same example $\ell_{\infty}\left(L_{2}\right)$, with essentially the same series $\sum_{n} f_{n}$, was given earlier in $[\mathrm{GvR},(4.3)]$. Expanding a remark made at the end of $[\mathrm{GvR}]$, we arrive at the following.

Example 7.1. There exists a $\sigma$-additive order bounded measure $\varphi: \mathcal{P}(\mathbb{N})$ $\rightarrow c\left(L_{2}\right)$ that has no absolute majorant. Here $c\left(L_{2}\right)$ denotes the closed separable sublattice of $\ell_{\infty}\left(L_{2}\right)$ consisting of convergent sequences in $L_{2}$.

Consider the series $\sum_{n} f_{n}$ described above. Note that the sums of all its subseries, and the order sum (o)- $\sum_{n}\left|f_{n}\right|=(1,1, \ldots)$, actually "sit" in $c\left(L_{2}\right)$. Thus $c\left(L_{2}\right)$ does not have Property (S). Let $\varphi: \mathcal{P}(\mathbb{N}) \rightarrow c\left(L_{2}\right)$ be the measure associated with the series $\sum_{n} f_{n}$. Evidently, it is $\sigma$ additive and order bounded. However, it does not have an absolute majorant $\nu: \mathcal{P}(\mathbb{N}) \rightarrow c\left(L_{2}\right)$. For, if such $\nu$ existed, it would be exhaustive, by Proposition 5.3 or [DU, Thm. I.4.2]. From this, as $\sum_{n \in J}\left|f_{n}\right| \leqslant \nu(J)$ for finite sets $J \subset \mathbb{N}$, it would follow that the series $\sum_{n}\left|f_{n}\right|$ is Cauchy. A contradiction.

As we will see in Theorem 7.7, the absence of Property $(\mathrm{S})$ is quite a typical phenomenon in some non-order continuous Banach lattices. Moreover, the basic ideas employed in the construction of the series $\sum_{n} f_{n}$ mentioned above, suitably modified, can also be used when dealing with those more general cases. After "discovering" [GvR] we realized that these ideas (employed also in Example 7.3) are in perfect accordance with the characterization of Banach lattices that are not order bounded $A M$, established in [GvR, Thm. 4.2].

But first, let us make a trivial observation that in our construction the "disjoint" blocks $\left(f_{n}: n \in J_{k}\right)$ consist of simple functions, and having realized that it is easy to give a sequential version of the construction - a solid Banach sequence space without Property (S).

Example 7.2. Let $\left(M_{k}\right)$ be a partition of $\mathbb{N}$ into consecutive intervals with $\left|M_{k}\right|=m_{k}=2^{n_{k}}$, where $n_{k} \rightarrow \infty$. Define $E$ to be the space of all real sequences $x=\left(t_{n}\right)$ such that

$$
\|x\|:=\sup _{k}\left(\frac{1}{m_{k}} \sum_{n \in M_{k}}\left|t_{n}\right|^{2}\right)^{1 / 2}<\infty,
$$

equipped with the norm given by this equality. Then $E$ is a Dedekind complete Banach lattice without Property (S). A required "bad" series 
can be constructed as described above using Rademacher-type functions on the intervals $M_{k}$.

Clearly, $E$ is Riesz isometric to the Banach lattice $F=\left(\sum_{k} \ell_{2}^{m_{k}}\right)_{\ell_{\infty}}$. Thus also $F$ fails to have Property (S). In fact, it is so for any $F$ defined in this way as soon as $\sup _{k} m_{k}=\infty$.

Next, we give a more direct extension of our original example $\ell_{\infty}\left(L_{2}\right)$ based on ideas quite different from those employed in [DW, Ex. 3] or $[\mathrm{GvR},(4.3)]$.

Example 7.3. If $E$ is a Banach lattice which is not Riesz isomorphic to an AM-space, then the Banach lattice $\ell_{\infty}(E)$ does not have Property (S).

As in the proof of Proposition 4.5, we can find an infinite sequence $\left(x_{n}\right)$ in $E$ such that for a certain partition $\left(M_{k}\right)$ of $\mathbb{N}$ into consecutive intervals,

$$
\begin{gathered}
\sup \left\{\left\|\sum_{n \in M_{k}} \varepsilon_{n} x_{n}\right\|_{E}: \varepsilon_{n}= \pm 1\right\} \leqslant 2^{-k} \text { and } \\
\left\|\sum_{n \in M_{k}}\left|x_{n}\right|\right\|_{E}=1, \quad k=1,2, \ldots
\end{gathered}
$$

Define a sequence $\left(\mathbf{x}_{n}\right)$ in $\ell_{\infty}(E)$ by setting $\mathbf{x}_{n}=\left(0, \ldots, 0, x_{n}, 0, \ldots\right)$ for $n \in M_{k}$, where $x_{n}$ occupies the $k$-th position, $k=1,2, \ldots$ Since

$$
\left\|\sum_{n \in M_{k}} \varepsilon_{n} \mathbf{x}_{n}\right\|_{\ell_{\infty}(E)}=\left\|\sum_{n \in M_{k}} \varepsilon_{n} x_{n}\right\|_{E} \leqslant 2^{-k} \quad \text { for } \varepsilon_{n}= \pm 1 \text { and every } k \text {, }
$$

the series $\sum_{n} \mathbf{x}_{n}$ is unconditionally convergent in $\ell_{\infty}(E)$. Moreover, denoting $u_{k}=\sum_{n \in M_{k}}\left|x_{n}\right|$, we have $\left\|u_{k}\right\|_{E}=1$ so that $\mathbf{u}:=\left(u_{k}\right) \in$ $\ell_{\infty}(E)$, and $\sum_{n=1}^{N}\left|\mathbf{x}_{n}\right| \leqslant \mathbf{u}$ for every $N$. Thus the series $\sum_{n} \mathbf{x}_{n}$ is order bounded in $\ell_{\infty}(E)$. However,

$$
\left\|\sum_{n \in M_{k}}\left|\mathbf{x}_{n}\right|\right\|_{\ell_{\infty}(E)}=\left\|\sum_{n \in M_{k}}\left|x_{n}\right|\right\|_{E}=1, \quad \text { for every } k,
$$

hence the series $\sum_{n} \mathbf{x}_{n}$ is not convergent in $\ell_{\infty}(E)$.

Remark 7.4. More generally, consider a sequence $\left(E_{n}\right)$ of Banach lattices that are not uniformly Riesz homeomorphic to AM-spaces. That 
is (see [APY, Thm. 3.1]), there is no finite constant $d$ such that condition $(*)$ stated in the proof of Proposition 4.5 holds for each of the spaces $E_{n}$. Then, by the same construction as above, the Banach lattice $\left(\sum_{n} E_{n}\right)_{\ell_{\infty}}$ does not have Property (S). In particular, in view of [APY, Thm. 2.1], this includes the Banach lattices constructed in Example 7.2 and, moreover, allows to replace the finite dimensional $\ell_{2}$-spaces used there with, for instance, finite dimensional $\ell_{p}$-spaces, $1 \leqslant p<\infty$. Of just this type is the earliest known example of a Banach lattice without Property (S), which was given in [G, Ex. 4.5, p. 95]: the $\ell_{\infty}$-sum of a sequence of finite dimensional $\ell_{1}$-spaces.

For every Banach lattice $E$, we define

$$
E_{A}=\left\{x \in E:|x| \geqslant x_{\alpha} \downarrow 0 \Longrightarrow\left\|x_{\alpha}\right\| \rightarrow 0\right\} .
$$

Thus $E_{A}$ is the largest ideal in $E$ on which the norm is order continuous, and $E_{A}$ is closed in $E$. For more information, see [W1].

We now proceed to the promised general result.

Sublemma 7.5. Let $F$ be a Banach lattice of measurable functions over a finite measure space $(X, \mathfrak{X}, \lambda)$. Let $g \in F_{A}$. Then $\left\|f_{n}\right\| \rightarrow 0$ for every sequence $\left(f_{n}\right)$ in $F$ such that $\left|f_{n}\right| \leqslant|g|$ and $\left\|f_{n}\right\|_{2} \rightarrow 0$. In other words, for every $\varepsilon>0$ there exists $\delta>0$ such that if $f \in F,|f| \leqslant|g|$ and $\|f\|_{2} \leqslant \delta$, then $\|f\| \leqslant \varepsilon$.

Proof. It is enough to note that $\left|f_{n}\right| \leqslant|g|$ implies that the $f_{n}$ 's have equi-absolutely continuous norms, and $\left\|f_{n}\right\|_{2} \rightarrow 0$ implies that $f_{n} \rightarrow 0$ in measure. As is well known, these two things assure that $\left\|f_{n}\right\| \rightarrow 0$.

Lemma 7.6. Let $E$ be an atomless Banach lattice. If $0<z \in E_{A}$, then for every $\varepsilon>0$ there exists a finite family $\left\{x_{i}: i \in I\right\}$ of elements in $E$ such that

$$
\left\|\sum_{j \in J} x_{j}\right\| \leqslant \varepsilon, \forall J \subset I \quad \text { and } \quad \sum_{i \in I}\left|x_{i}\right|=z .
$$

Proof. Consider the closed ideal $E_{z} \subset E_{A}$ generated by z. By [LT, Thm. 1.b.14], $E_{z}$ is order isometric to a solid order continuous Banach lattice $F$ of measurable functions over some nonatomic probability measure space $(X, \mathfrak{X}, \lambda)$, with $z$ corresponding to the constant function 1 on 
$X$. Thus it is enough to show the existence of a finite family $\left\{g_{i}: i \in I\right\}$ in $F$ such that

$$
\left\|\sum_{j \in J} g_{j}\right\| \leqslant \varepsilon, \forall J \subset I \quad \text { and } \quad \sum_{i \in I}\left|g_{i}\right|=1 .
$$

Fix a $\delta>0$ provided by Sublemma 7.5 for the given number $\varepsilon$ and the function $g=1$. Choose an integer $N>\delta^{-2}$, denote $I=\{1, \ldots, N\}$, and let $\rho_{i}$ for $i \in I$ be the first $N$ Rademacher-type functions on $(X, \mathfrak{X}, \lambda)$. Set $g_{i}=N^{-1} \rho_{i}$ for $i \in I$. Then, for any subset $J$ of $I$,

$$
\left|\sum_{j \in J} g_{j}\right| \leqslant 1 \quad \text { and } \quad \int_{X}\left|\sum_{j \in J} g_{j}\right|^{2} d \lambda=|J| \cdot N^{-2} \leqslant N^{-1} \leqslant \delta^{2} \text {, }
$$

and, in view of the sublemma, it follows easily that the $g_{i}$ 's are as required.

Theorem 7.7. If $E$ is an atomless Banach lattice in which $E_{A}$ is not a band, then $E$ lacks Property (S). In fact, there exists an unconditionally convergent series $\sum_{n} x_{n}$ in $E_{A}$ that is order bounded in $E$ and for which the series $\sum_{n}\left|x_{n}\right|$ does not converge.

Proof. Without loss of generality we may assume that $E_{A}$ is order dense in $E$ (replacing, if necessary, $E$ by $E^{d d}$; cf. [AB1, Thm. 1.11], [AB2, Thm. 3.4]). Fix $z \in E_{+} \backslash E_{A}$. By [W1, Lemma 3], there exists a disjoint sequence $\left(z_{k}\right)$ in $E_{A}$ such that $0 \leqslant z_{k} \leqslant z$ and $\inf _{k}\left\|z_{k}\right\|>0$. Applying Lemma 7.6, we find a partition $\left(I_{k}\right)$ of $\mathbb{N}$ into consecutive intervals and, for each $k$, a family $\left\{x_{n}: n \in I_{k}\right\}$ of elements in $E$ such that

$$
\left\|\sum_{n \in J} x_{n}\right\| \leqslant 2^{-k}, \quad \forall J \subset I_{k} \quad \text { and } \quad \sum_{n \in I_{k}}\left|x_{n}\right|=z_{k} .
$$

Evidently, the series $\sum_{n} x_{n}$ is unconditionally convergent and order bounded, but the series $\sum_{n}\left|x_{n}\right|$ is not convergent.

Remarks 7.8. (a) Theorem 7.7 does not extend to atomic (or discrete) Banach lattices. Indeed, $\left(\ell_{\infty}\right)_{A}=c_{0}$ is not a band in $\ell_{\infty}$, and yet $\ell_{\infty}$ has Property (S). On the other hand, as we saw in Example 7.2 (see also 
the remark after Example 7.3), all Banach lattices $F=\left(\sum_{k} \ell_{2}^{m_{k}}\right)_{\ell_{\infty}}$, where $m_{k} \rightarrow \infty$, fail to have Property (S), and for each of them $F_{A}=$ $\left(\sum_{k} \ell_{2}^{m_{k}}\right)_{c_{0}}$ is not a band. (In fact, for a solid Banach lattice $F \subset \omega=$ $\mathbb{R}^{\mathbb{N}}, F_{A}$ is not a band in $F$ unless $F_{A}=F$.) We do not know, however, whether all Orlicz sequence spaces $\ell_{\varphi}$ have Property (S).

(b) If $E$ is a Banach lattice and $E_{A}$ is a band (it is so, in particular, when $E_{A}=\{0\}$ ), then $E$ may or may not have Property (S). We illustrate this statement by the following examples.

Let $F:=L_{2}(0, \infty) \cap L_{\infty}(0, \infty)$ with the norm $\|f\|=\max \left(\|f\|_{2},\|f\|_{\infty}\right)$. Then $F$ is Dedekind complete, has the Fatou property, $F_{A}=\{0\}$, and $F$ is not isomorphic to an AM-space. Nevertheless, as both $L_{2}(0, \infty)$ and $L_{\infty}(0, \infty)$ have Property (S), so does $F$. However, if $E:=\ell_{\infty}(F)$, then $E_{A}=\{0\}$ and, by Example 7.3, $E$ lacks Property (S).

Likewise, if $G:=L_{\infty}[0,1]$ and $H:=\ell_{2}(G)$, then $H_{A}=\{0\}$ and, as easily seen, $H$ has Property (S). However, $H$ is not isomorphic to an AM-space so that, by Example $7.3, \ell_{\infty}(H)$ lacks Property $(\mathrm{S})$ while $\ell_{\infty}(H)_{A}=\{0\}$.

Proposition 7.9. Let $E$ be a $\sigma$-Dedekind complete Banach lattice without Property (S). If the $\sigma$-algebra $\Sigma$ admits an atomless probability measure $m$, then there exists an atomless order bounded $\sigma$-additive measure $\mu: \Sigma \rightarrow E$ with a non- $\sigma$-additive proper modulus $|\mu|$.

Proof. Take an unconditionally convergent and perfectly order bounded series $\sum_{n} x_{n}$ in $E$ such that the series $\sum_{n}\left|x_{n}\right|$ is not convergent. Next, fix an infinite disjoint sequence $\left(A_{n}\right)$ in $\Sigma$ with all $a_{n}:=m\left(A_{n}\right)>0$, and define (mutually singular) atomless probability measures $m_{n}$ on $\Sigma$ by $m_{n}(A)=a_{n}^{-1} m_{n}\left(A \cap A_{n}\right)$. Finally, define a measure $\mu: \Sigma \rightarrow E$ by $\mu(A)=\sum_{n=1}^{\infty} m_{n}(A) x_{n}$. Obviously, $\mu$ is $\sigma$-additive, order bounded, and atomless. Since $E$ is $\sigma$-Dedekind complete, the modulus $|\mu|$ exists. In fact, as easily seen, $|\mu|(A)=(\mathrm{o})-\sum_{n} a_{n}^{-1} m\left(A \cap A_{n}\right)\left|x_{n}\right|$ for every $A \in \Sigma$. However, $|\mu|$ is not $\sigma$-additive because $|\mu|\left(A_{n}\right)=\left|x_{n}\right|$ for every $n$.

We conclude this section with yet another example of a $\sigma$-additive measure with a proper non- $\sigma$-additive modulus. It also shows that the assumption that $E$ is Fatou can be removed from neither Proposition 6.1 nor Theorem 6.2, and that Property (S) alone is not enough for the transition of $\sigma$-additivity from a measure to its modulus. The example below is, essentially, only a slightly modified version of Example 5.14 on 
p. 106 (see also an observation concerning it on p. 112) in [G] (cf. also $[\mathrm{GvR},(3.4)])$. Since, however, $[\mathrm{G}]$ is not easily available, we give it in extenso.

Example 7.10. A $\sigma$-additive order bounded measure $\mu$ with values in a Dedekind complete AM-space whose modulus $|\mu|$ is not exhaustive and, a fortiori, not $\sigma$-additive.

For each $n \in \mathbb{N}$, let $E_{n}=\left(\ell_{\infty},\|\cdot\|_{n}\right)$, where $\|x\|_{n}=\max \left\{\|x\|_{\infty}\right.$, $\left.n \lim \sup _{k}\left|t_{k}\right|\right\}$ for $x=\left(t_{k}\right) \in \ell_{\infty}$. Clearly, $E_{n}$ is an AM-space, $\|x\|_{n}=$ $\|x\|_{\infty}$ for $x \in c_{0}$, and $0 \leqslant x_{j} \uparrow x$ in $E_{n}$ implies $\|x\|_{n} \leqslant n \lim _{j}\left\|x_{j}\right\|_{n}$, where the constant factor $n$ is best possible. Let $E=\left(E_{1} \oplus E_{2} \oplus \ldots\right)_{\ell_{\infty}}$. Then $E$ is a Dedekind complete AM-space without the Fatou property, and it can be viewed as an ideal in $\omega=\mathbb{R}^{\mathbb{N}}$.

Recall that we denote by $\mathcal{B}$ the Borel $\sigma$-algebra on the interval $[0,1]$. Consider a measure $\mu=\left(\mu_{n}\right): \mathcal{B} \rightarrow E$, where each $\mu_{n}: \mathcal{B} \rightarrow E_{n}$ is defined by

$$
\mu_{n}(A)=\frac{2^{n}}{n}\left(\int_{A \cap I_{n}} r_{k} d \lambda\right)_{k \in \mathbb{N}}
$$

and $I_{n}:=\left(2^{-n}, 2^{-n+1}\right)$. Note that $\mu_{n}$ is $c_{0}$-valued so that

$$
\left\|\mu_{n}(A)\right\|_{n}=\left\|\mu_{n}(A)\right\|_{\infty}=\frac{2^{n}}{n} \sup _{k}\left|\int_{A \cap I_{n}} r_{k} d \lambda\right| \leqslant \frac{2^{n}}{n} \lambda\left(A \cap I_{n}\right) \leqslant \frac{1}{n} .
$$

From this it follows that each $\mu_{n}$ is $\sigma$-additive and that $\mu(A) \in\left(E_{1} \oplus\right.$ $\left.E_{2} \oplus \ldots\right)_{c_{0}}$ for all $A \in \mathcal{B}$. Moreover, for any $m \in \mathbb{N}$,

$$
\begin{gathered}
\|\mu(A)\|=\sup _{n}\left\|\mu_{n}(A)\right\|_{n} \leqslant \sup _{n} \frac{2^{n}}{n} \lambda\left(A \cap I_{n}\right) \leqslant \\
\max \left\{\sup _{1 \leqslant n \leqslant m} \frac{2^{n}}{n} \lambda\left(A \cap I_{n}\right), \frac{1}{m}\right\} .
\end{gathered}
$$

Hence $\left\|\mu\left(A_{j}\right)\right\| \rightarrow 0$ whenever $A_{j} \downarrow \emptyset$ in $\mathcal{B}$. Thus $\mu$ assumes its values in $E$ and is $\sigma$-additive. Next, for every $A \in \mathcal{B}$ and $n \in \mathbb{N}$,

$\left|\mu_{n}\right|(A)=\frac{2^{n}}{n}\left(\lambda\left(A \cap I_{n}\right), \lambda\left(A \cap I_{n}\right), \ldots\right) \quad$ and $\quad\left\|\left|\mu_{n}\right|(A)\right\|_{n}=2^{n} \lambda\left(A \cap I_{n}\right)$.

By Corollary 1.8 , the modulus $|\mu|$ exists properly and $|\mu|=\left(\left|\mu_{n}\right|\right)$. Since $\left\||\mu|\left(I_{j}\right)\right\|=2^{j} \lambda\left(I_{j}\right)=1$ for all $j$, we see that $|\mu|$ is not exhaustive. 


\section{Remarks on the proper existence of the mod- ulus of a measure}

In this section, unless stated otherwise, $E$ is assumed to be an arbitrary Riesz space.

We first show that if a measure admits a Hahn decomposition, then it has a proper modulus. In the proposition below, measures $\mu_{1}, \mu_{2}: \mathcal{A} \rightarrow$ $E$ are called mutually singular if there is $A_{0} \in \mathcal{A}$ such that $\mu_{1}\left(\mathcal{A} \cap A_{0}\right)=$ $\mu_{2}\left(\mathcal{A} \cap\left(S \backslash A_{0}\right)\right)=\{0\}$.

Proposition 8.1. For a measure $\mu: \mathcal{A} \rightarrow E$, the following are equivalent:

(a) $\mu(\mathcal{A})$ has a largest element.

(b) $\mu$ admits a Hahn decomposition: There exist mutually singular positive measures $\mu_{1}, \mu_{2}: \mathcal{A} \rightarrow E$ such that $\mu=\mu_{1}-\mu_{2}$.

Moreover, if (b) holds, then $|\mu|$ exists properly and $|\mu|=\mu_{1}+\mu_{2}$.

Proof. Let $A_{0} \in \mathcal{A}$ be such that $\mu(A) \leqslant \mu\left(A_{0}\right)$ for all $A \in \mathcal{A}$. If $B \subset A_{0}$ and $C \subset S \backslash A_{0}$, then $\mu\left(A_{0}\right)-\mu(B) \leqslant \mu\left(A_{0}\right)$ and $\mu(C)+\mu\left(A_{0}\right) \leqslant \mu\left(A_{0}\right)$, hence $\mu(B) \geqslant 0$ and $\mu(C) \leqslant 0$. It follows that the measures $\mu_{1}$ and $\mu_{2}$ defined by $\mu_{1}(A)=\mu\left(A \cap A_{0}\right)$ and $\mu_{2}(A)=-\mu\left(A \backslash A_{0}\right)$ are as required in (b).

Conversely, let $\mu_{1}, \mu_{2}$ satisfy (b) and let $A_{0}$ be as required in the definition of their mutual singularity. Then, for every $A \in \mathcal{A}, \mu(A) \leqslant$ $\mu_{1}(A) \leqslant \mu_{1}\left(A_{0}\right)=\mu\left(A_{0}\right)$ so that $\mu\left(A_{0}\right)$ is the largest element in $\mu(\mathcal{A})$. Moreover, if $\pi \in \Pi(A)$ and $\pi$ is finer than the partition $\left\{A \cap A_{0}, A \backslash A_{0}\right\}$ of $A$, then one easily verifies that $\sum_{D \in \pi}|\mu(D)|=\mu_{1}(A)+\mu_{2}(A)$. It follows that $|\mu|(A)=\mu_{1}(A)+\mu_{2}(A)$ and that $|\mu|$ exists properly.

We next present some results concerning the proper existence of the modulus $|\mu|$ of an order bounded measure $\mu$ when it is a priori known that $|\mu|$ exists.

Proposition 8.2. If the modulus $|\mu|$ of a measure $\mu: \mathcal{A} \rightarrow E$ exists, then the class of all sets $A \in \mathcal{A}$ on which it exists properly is an ideal in $\mathcal{A}$.

Proof. Denote the class of such sets $A$ by $\mathcal{A}^{\prime}$. We first show that if $B \in \mathcal{A}$ and $B \subset A \in \mathcal{A}^{\prime}$, then $B \in \mathcal{A}^{\prime}$. Suppose $u \in E$ is such that 
$\sum_{D \in \pi}|\mu(D)| \leqslant u$ for all $\pi \in \Pi(B)$. Then for every $\pi \in \Pi(A)$,

$$
\sum_{D \in \pi}|\mu(D)| \leqslant \sum_{D \in \pi}|\mu(D \cap B)|+\sum_{D \in \pi}|\mu(D \cap(A \backslash B))| \leqslant u+|\mu|(A \backslash B) .
$$

It follows that $|\mu|(B)+|\mu|(A \backslash B) \leqslant u+|\mu|(A \backslash B)$. In consequence, $|\mu|(B) \leqslant u$ which means that $|\mu|(B)=\sup \left\{\sum_{D \in \pi}|\mu(D)|: \pi \in \Pi(B)\right\}$ so that $B \in \mathcal{A}^{\prime}$.

It remains to show if $A, B \in \mathcal{A}^{\prime}$, then $A \cup B \in \mathcal{A}^{\prime}$. In view of what we have proved above it can be assumed that $A \cap B=\emptyset$, and then a verification that $|\mu|$ exists properly on $A \cup B$ is straightforward.

Corollary 8.3. If the modulus $|\mu|$ of a measure $\mu: \mathcal{A} \rightarrow E$ exists, and if it exists properly on $S$, then $|\mu|$ exists properly.

A measure $\mu: \mathcal{A} \rightarrow E_{+}$is said to be order-countably additive, or $(o)$ $\sigma$-additive, if $\mu(A)=(\mathrm{o})-\sum_{n} \mu\left(A_{n}\right):=(\mathrm{o})-\lim _{n} \sum_{i=1}^{n} \mu\left(A_{i}\right)$ whenever $A \in \mathcal{A}$ and $\left(A_{n}\right)$ is a disjoint sequence in $\mathcal{A}$ with union $A$; equivalently, $\mu(A)=(\mathrm{o})-\lim _{n} \mu\left(A_{n}\right)$ whenever $A_{n} \uparrow A$ in $\mathcal{A}$.

Proposition 8.4. If the modulus $|\mu|$ of a measure $\mu: \Sigma \rightarrow E$ exists and is (o)- $\sigma$-additive, then the class of all sets $A \in \Sigma$ on which $|\mu|$ exists properly is a $\sigma$-ideal in $\Sigma$.

Proof. Denote the class of such sets $A$ by $\Sigma^{\prime}$. In view of Proposition 8.2 we only have to verify that for every increasing sequence $\left(A_{n}\right)$ in $\Sigma^{\prime}$ its union $A$ is also in $\Sigma^{\prime}$.

Take any $x \in E_{+}$such that $\sum_{D \in \pi}|\mu(D)| \leqslant x$ for each $\pi \in \Pi(A)$. From this, as the $A_{n}$ 's are in $\Sigma^{\prime}$, it follows that $|\mu|\left(A_{n}\right) \leqslant x$ for all $n$. Since $|\mu|$ is $(o)$ - $\sigma$-additive, $|\mu|(A)=(0)-\lim _{n}|\mu|\left(A_{n}\right) \leqslant x$. Therefore, $|\mu|(A)=\sup \left\{\sum_{D \in \pi}|\mu(D)|: \pi \in \Pi(A)\right\}$.

Remark. According to Proposition 8.6 below, if $E$ is a $\sigma$-Dedekind complete TRS and $\mu: \Sigma \rightarrow E$ is a $\sigma$-additive measure whose modulus $|\mu|$ exists, then $|\mu|$ is $(o)$ - $\sigma$-additive.

Corollary 8.5. Let $\mu: \Sigma \rightarrow E$ be a measure such that the modulus $|\mu|$ exists and is (o)- $\sigma$-additive. Also, assume that every disjoint family of non- $\mu$-null sets in $\Sigma$ is countable. Then there exists $S_{0} \in \Sigma$ such that $|\mu|$ exists properly on every set $A \in \Sigma$ contained in $S_{0}$, and does not exist properly on any non- $\mu$-null set $A \in \Sigma$ that is disjoint from $S_{0}$. 
Proof. Take a maximal disjoint family $\mathcal{D}$ of non- $\mu$-null sets on which $|\mu|$ exists properly. By assumption, it is countable, and its union, $S_{0}$, is as required, by Proposition 8.4 and maximality of $\mathcal{D}$.

The following result has the same proof as Proposition 6.4, replacing lim in the definition of $\nu$ with (o)- $\lim$.

Proposition 8.6. Let $E$ be $a$ TRS and $\mu: \mathcal{A} \rightarrow E$ a $\sigma$-additive measure such that the modulus $|\mu|$ exists. If for every increasing sequence $\left(A_{n}\right)$ in $\mathcal{A}$ the order limit (o)- $\lim _{n}|\mu|\left(A_{n}\right)$ exists in $E$ (it is so e.g. when $E$ is $\sigma$-Dedekind complete), then $|\mu|$ is (o)- $\sigma$-additive.

Note that if $|\mu|$ is $\sigma$-additive, then it is also (o)- $\sigma$-additive.

We are now going to show that there is an intimate relation between the problem of the proper existence of the modulus $|\mu|$ of a measure $\mu$, and a similar problem concerning the induced integral operator $I_{\mu}$. The latter problem, in its general form, can be formulated as follows (see [AB2, Chap 1, Sec. 1] and [vR, Problem 1.2] for more information):

Let $E$ and $F$ be Riesz spaces, and let $T: E \rightarrow F$ be a regular operator; that is, $T$ admits an absolute majorant, a positive operator $P: E \rightarrow F$ with $|T(x)| \leqslant P(x)$ for all $x \in E_{+}$. The smallest of such majorants for $T$, if exists, is called the modulus of $T$ and denoted $|T|$. If $\sup T([-x, x])$ exists in $F$ for each $x \in E_{+}$, then the modulus $|T|$ exists and $|T|(x)=\sup T([-x, x])$ for all $x \in E_{+}$; that is, $|T|$ exists properly. However, it is unknown whether the mere existence of the modulus $|T|$ always implies its proper existence.

In Proposition 8.8 below we show that, in particular, if $E$ is a Banach lattice, then for an order bounded measure $\mu: \mathcal{A} \rightarrow E$ and the induced integral operator $I_{\mu}: B(\mathcal{A}) \rightarrow E$, the questions of proper existence of $|\mu|$ and $\left|I_{\mu}\right|$ are equivalent. Here, following [DU, p. 6], we denote by $B(\mathcal{A})=B(S, \mathcal{A})$ the Banach lattice consisting of functions $f: S \rightarrow \mathbb{R}$ that are uniform limits of $(\mathcal{A}-)$ simple functions, equipped with the sup norm.

Actually, we will work in the more general setting where $E$ is an arbitrary Riesz space that is relatively uniformly complete. Recall that the latter means that for every $0 \leqslant u \in E$ the ideal $E_{u}$ generated by $u$ is a Banach space under the norm $\|\cdot\|_{u}$ whose closed unit ball $=[-u, u]$. We denote by $o b a(\mathcal{A}, E)$ and $L_{b}(B(\mathcal{A}), E)$ the ordered vector spaces of all order bounded measures $\mu: \mathcal{A} \rightarrow E$ and all order bounded operators 
$T: B(\mathcal{A}) \rightarrow E$, respectively. For every $\mu \in \operatorname{oba}(\mathcal{A}, E)$ and $f \in B(\mathcal{A})$, let $I_{\mu}(f)=\int_{S} f d \mu$. This makes sense because choosing $0 \leqslant u \in E$ so that $\mu(\mathcal{A}) \subset[-u, u]$, we may view $\mu$ as a bounded measure with values in the Banach space $\left(E_{u},\|\cdot\|_{u}\right)$, and then define the integral $\int_{S} f d \mu$ in the usual way (see e.g. [DU, p. 6]). Note that the operator $I_{\mu}: B(\mathcal{A}) \rightarrow\left(E_{u},\|\cdot\|_{u}\right)$ is continuous.

In the final stage of the proof of Proposition 8.8 we will need the following (certainly well known)

Lemma 8.7. For every $0 \leqslant f \in B(\mathcal{A})$ there is a sequence $\left(f_{n}\right)$ of simple functions such that $0 \leqslant f_{n}(s) \uparrow f(s)$ for all $s \in S$ and $\left\|f-f_{n}\right\| \rightarrow 0$.

Proof. Given any $\varepsilon>0$, there exists a simple function $g=\sum_{i=1}^{n} a_{i} 1_{A_{i}}$ with disjoint $A_{i} \in \mathcal{A}$, such that $\|f-g\| \leqslant \varepsilon$. Thus $a_{i}-\varepsilon \leqslant f(s) \leqslant a_{i}+\varepsilon$ for $s \in A_{i}, i=1, \ldots, n$. Let $b_{i}=\max \left\{a_{i}-\varepsilon, 0\right\}$. Then $h=\sum_{i=1}^{n} b_{i} 1_{A_{i}}$ is a simple function and $0 \leqslant h \leqslant f$. Moreover, if $s \in A_{i}$, then $0 \leqslant$ $f(s)-h(s)=f(s)-b_{i} \leqslant\left(a_{i}+\varepsilon\right)-b_{i}$. Observe that $\left(a_{i}+\varepsilon\right)-b_{i}=$ $\left(a_{i}+\varepsilon\right)-\left(a_{i}-\varepsilon\right)=2 \varepsilon$ when $b_{i}=a_{i}-\varepsilon \geqslant 0$; and $\left(a_{i}+\varepsilon\right)-b_{i}=a_{i}+\varepsilon<2 \varepsilon$ when $b_{i}=0$, because then $a_{i}-\varepsilon<0$, or $a_{i}<\varepsilon$. It follows that $0 \leqslant f-h \leqslant 2 \varepsilon$ on $S$.

Applying the above with $\varepsilon=(2 k)^{-1}$ gives a sequence $\left(h_{k}\right)$ of simple functions such that $0 \leqslant f-h_{k} \leqslant 1 / k$. Then, setting $f_{n}=h_{1} \vee \cdots \vee h_{n}$, we get an increasing sequence $\left(f_{n}\right)$ of simple functions such that $0 \leqslant f_{n} \leqslant f$ and $f_{n} \rightarrow f$ uniformly.

Proposition 8.8. Let $E$ be a relatively uniformly complete Riesz space. Then the map $\mu \rightarrow I_{\mu}$ is a linear and order isomorphism between the ordered vector spaces oba $(\mathcal{A}, E)$ and $L_{b}(B(\mathcal{A}), E)$. Moreover, if $\mu \in$ oba $(\mathcal{A}, E)$, then

(a) $\mu$ admits an absolute majorant iff $I_{\mu}$ admits an absolute majorant;

(b) $|\mu|$ exists iff $\left|I_{\mu}\right|$ exists, and if this is the case, then $\left|I_{\mu}\right|=I_{|\mu|}$;

(c) the modulus $|\mu|$ exists properly iff the modulus $\left|I_{\mu}\right|$ exists properly.

Proof. Only part (c) requires a proof. Note: Below, we will use continuity of $I_{\mu}$ and $I_{|\mu|}$ as operators from $B(\mathcal{A})$ to $\left(E_{u},\|\cdot\|_{u}\right)$ for suitably chosen $u$ 's (not indicated explicitly).

For each $A \in \mathcal{A}$ denote $M_{A}=\left\{\sum_{D \in \pi}|\mu(D)|: \pi \in \Pi(A)\right\}$. 
Suppose $\left|I_{\mu}\right|=I_{|\mu|}$ exists properly. Thus

$$
\left|I_{\mu}\right|(f)=\sup \left\{\left|I_{\mu}(g)\right|:|g| \leqslant f\right\}=\sup \left\{I_{\mu}(g):|g| \leqslant f\right\}
$$

for all $0 \leqslant f \in B(\mathcal{A})$.

Fix $A \in \mathcal{A}$ and let $x$ be an upper bound of $M_{A}$. If $h \in B(\mathcal{A})$ is simple and $|h| \leqslant 1_{A}$, then clearly $\left|I_{\mu}(h)\right| \leqslant x$. If $g \in B(\mathcal{A})$ is arbitrary and $|g| \leqslant 1_{A}$, then choose sequences $\left(u_{n}\right),\left(v_{n}\right)$ of simple functions so that $0 \leqslant u_{n}, v_{n} \leqslant 1_{A}$ and $u_{n} \uparrow g^{+}, v_{n} \uparrow g^{-}$uniformly. Then $\left|u_{n}-v_{n}\right| \leqslant 1_{A}$, hence $\left|I_{\mu}\left(u_{n}-v_{n}\right)\right| \leqslant x$, and the continuity of $I_{\mu}$ gives $\left|I_{\mu}(g)\right| \leqslant x$. Therefore $|\mu|(A)=I_{|\mu|}\left(1_{A}\right)=\left|I_{\mu}\right|\left(1_{A}\right) \leqslant x$. In consequence, $|\mu|(A)=$ $\sup M_{A}$; that is, $|\mu|(A)$ exists properly.

Suppose now that $|\mu|$ exists properly. Thus $|\mu|(A)=\sup M_{A}$ for every $A \in \mathcal{A}$. By (b), $\left|I_{\mu}\right|$ exists and $\left|I_{\mu}\right|=I_{|\mu|}$. Hence, for every $A \in \mathcal{A},|\mu|(A)=\left|I_{\mu}\right|\left(1_{A}\right)$ is an upper bound of the set $\left\{\left|I_{\mu}(g)\right|:|g| \leqslant\right.$ $\left.1_{A}\right\}$. In fact, it is its smallest upper bound because $\sum_{i}\left|\mu\left(D_{i}\right)\right|=$ $\sup \left\{\left|I_{\mu}\left(\sum_{i} \varepsilon_{i} 1_{D_{i}}\right)\right|: \varepsilon_{i}= \pm 1\right\}$ for all $\left\{D_{1}, \ldots, D_{n}\right\} \in \Pi(A)$. It follows that $(*)$ holds for $f=1_{A}$, hence also for $f=a 1_{A}$, where $a \in \mathbb{R}_{+}$.

Now, take any simple function $f=\sum_{i=1}^{n} a_{i} 1_{A_{i}}$, where $a_{i} \geqslant 0$ and $A_{i} \in \mathcal{A}$ are disjoint. Note that $\{g:|g| \leqslant f\}=\sum_{i=1}^{n}\left\{g_{i}:\left|g_{i}\right| \leqslant a_{i} 1_{A_{i}}\right\}$ and that, by the preceding part, $\sup \left\{I_{\mu}\left(g_{i}\right):\left|g_{i}\right| \leqslant a_{i} 1_{A_{i}}\right\}$ exists and equals $\left|I_{\mu}\right|\left(a_{i} 1_{A_{i}}\right)$ for $i=1, \ldots, n$. Hence $\sup \left\{I_{\mu}(g):|g| \leqslant f\right\}$ exists and equals $\sum_{i=1}^{n}\left|I_{\mu}\right|\left(a_{i} 1_{A_{i}}\right)=\left|I_{\mu}\right|(f)$. Thus $(*)$ holds for nonnegative simple functions $f \in B(\mathcal{A})$.

Finally, let $0 \leqslant f \in B(\mathcal{A})$ be arbitrary. By the definition of $\left|I_{\mu}\right|$, $\left|I_{\mu}\right|(f)$ is an upper bound of the set $\left\{I_{\mu}(g):|g| \leqslant f\right\}$. Let $x$ be any of its upper bounds. Applying Lemma 8.7, choose a sequence $\left(f_{n}\right)$ of positive simple functions uniformly increasing to $f$. Clearly, $x$ is an upper bound of each set $\left\{I_{\mu}(g):|g| \leqslant f_{n}\right\}$ and so $\left|I_{\mu}\right|\left(f_{n}\right) \leqslant x$. By the continuity of $\left|I_{\mu}\right|,\left|I_{\mu}\right|(f) \leqslant x$. Therefore, $\left|I_{\mu}\right|(f)=\sup \left\{I_{\mu}(g):|g| \leqslant f\right\}$. This completes the proof.

\section{$9 \quad$ Absolutely exhaustive operators}

We shall say that a continuous linear operator $T$ from $B(\mathcal{A})$ to a TVS $E$ is exhaustive (see $[\mathrm{K}])$ if, for every bounded sequence $\left(f_{n}\right)$ in $B(\mathcal{A})$ consisting of disjoint (= disjointly supported) functions, $T\left(f_{n}\right) \rightarrow 0$ or, equivalently, the series $\sum_{n} T\left(f_{n}\right)$ is (unconditionally) Cauchy. (For 
$E$ a Banach space this is equivalent to $T$ being weakly compact.) If $E$ is a TRS and $T$ satisfies a stronger requirement that, for all $\left(f_{n}\right)$ as above, the series $\sum_{n}\left|T\left(f_{n}\right)\right|$ is Cauchy, then we shall say that $T$ is absolutely exhaustive. Evidently, if $T$ has an exhaustive absolute majorant $P: B(\mathcal{A}) \rightarrow E$, then $T$ is absolutely exhaustive.

Proposition 9.1. Let $E$ be a TRS. A continuous linear operator $T: B(\mathcal{A}) \rightarrow E$ is absolutely exhaustive iff the associated (bounded) measure $\mu_{T}: \mathcal{A} \rightarrow E$, defined by $\mu_{T}(A)=T\left(1_{A}\right)$, is absolutely exhaustive.

Proof. Denote by $W$ the solid hull of the image by $T$ of the closed unit ball in $B(\mathcal{A})$. Note that $W$ is a bounded subset of $E$.

Let $\mu_{T}$ be absolutely exhaustive. Take a bounded disjoint sequence $\left(g_{n}\right) \subset B(\mathcal{A})$ consisting of simple functions. We may assume that $\left\|g_{n}\right\|_{\infty} \leqslant 1$ for each $n$. Then, denoting by $A_{n}$ the support of $g_{n}$, we have $g_{n}=\sum_{j \in J_{n}} c_{j} 1_{C_{j}}$, where $\left(J_{n}\right)$ is a partition of $\mathbb{N}$ into a sequence of consecutive intervals, $\left\{C_{j}: j \in J_{n}\right\}$ is an $\mathcal{A}$-partition of $A_{n}$, and $\left|c_{j}\right| \leqslant 1$. Clearly, $\left|T\left(g_{n}\right)\right| \leqslant \sum_{j \in J_{n}}\left|\mu_{T}\left(C_{j}\right)\right|$. Since, by assumption, the series $\sum_{j}\left|\mu_{T}\left(C_{j}\right)\right|$ is Cauchy, it follows that so is the series $\sum_{n}\left|T\left(g_{n}\right)\right|$.

Now, let $\left(f_{n}\right)$ be an arbitrary bounded and disjoint sequence in $B(\mathcal{A})$. Fix any solid zero-neighborhood $V=V_{0}$ in $E$, and next choose a sequence $\left(V_{n}\right)$ of solid zero-neighborhoods in $E$ so that $V_{n}+V_{n} \subset V_{n-1}$ for all $n \geqslant 1$. Furthermore, for each $n \geqslant 1$ choose $\alpha_{n}>0$ such that $\alpha_{n} W \subset V_{n}$. By Lemma 8.7 and the continuity of $T$, we find a sequence $\left(g_{n}\right)$ of simple functions such that $\left|g_{n}\right| \leqslant\left|f_{n}\right|$ and $\left|T\left(f_{n}-g_{n}\right)\right| \in \alpha_{n} W$ for every $n$. Of course, the sequence $\left(g_{n}\right)$ is bounded and disjoint. Note that ||$T\left(f_{n}\right)|-| T\left(g_{n}\right)|| \leqslant\left|T\left(f_{n}-g_{n}\right)\right| \in \alpha_{n} W$, hence $\left|T\left(f_{n}\right)\right| \leqslant$ $\left|T\left(g_{n}\right)\right|+\alpha_{n} w_{n}$ for some $w_{n} \in W$. Since, by the first part of the proof, the series $\sum_{n}\left|T\left(g_{n}\right)\right|$ is Cauchy, there is $k$ such that $\sum_{n=k}^{m}\left|T\left(g_{n}\right)\right| \in V_{1}$ for $m \geqslant k$. Now, if $m \geqslant k$, then

$$
\sum_{n=k}^{m}\left|T\left(f_{n}\right)\right| \leqslant \sum_{n=k}^{m}\left|T\left(g_{n}\right)\right|+\sum_{n=k}^{m} \alpha_{n} w_{n} \in V_{1}+\sum_{n=k}^{m} V_{n} \subset V_{1}+V_{k-1} \subset V_{0}=V,
$$

and it follows that the series $\sum_{n}\left|T\left(f_{n}\right)\right|$ is Cauchy.

The other direction is obvious.

Corollary 9.2. Let $E$ be a Banach lattice with the Fatou property, and $T: B(\mathcal{A}) \rightarrow E$ an order bounded linear operator such that the modu- 
lus $|T|$ exists properly. Then $|T|$ is weakly compact iff $T$ is absolutely exhaustive.

Proof. By Proposition 8.8, $T=I_{\mu}$ for some $\mu \in o b a(\mathcal{A}, E)$, and the modulus $|\mu|$ exists properly. Now, by Propositions 9.1 and 6.1 (a), and in view of [DU, Thm. VI.1.1], we have the following chain of equivalences: $T$ is absolutely exhaustive iff $\mu$ is absolutely exhaustive iff $|\mu|$ is exhaustive iff $|T|=I_{|\mu|}$ is weakly compact.

Similarly, using Theorem 6.2 one obtains the following.

Corollary 9.3. Let $E$ be a Banach lattice with the Fatou property and Property $(\mathrm{S})$, and $T: B(\mathcal{A}) \rightarrow E$ an order bounded weakly compact linear operator. If the modulus $|T|$ exists properly, then it is weakly compact.

Remark. Results analogous to those stated above also hold when $B(\mathcal{A})$ is replaced with a space $L_{\infty}(\lambda)$ of bounded measurable functions. For other results dealing with the question of weak compactness of $|T|$, see [G, Thm. 6.4, p. 112], [GvR], [CW].

\section{References}

[APY] Y. A. Abramovič, E. D. Positselskiı̌ and L. P. Yanovskiǔ, On some parameters associated with normed lattices and on series characterisation of M-spaces, Studia Math. 63 (1978), 1-8.

[AB1] C. Aliprantis and O. Burkinshaw, Locally solid Riesz spaces, Pure and Applied Mathematics Series, No. 76, Academic Press, New York and London, 1978.

[AB2] , Positive operators, Pure and Applied Mathematics Series, No. 119, Academic Press, New York, 1985.

[CL] D. I. Cartwright and H. P. Lotz, Some characterizations of AM- and AL-spaces, Math. Z. 142 (1975), 97-103.

[CW] Z. L. Chen and A. W. Wickstead, Vector lattices of weakly compact operators on Banach lattices, Trans. Amer. Math. Soc. 352 (1999), 397-412.

[C] C. Constantinescu, Spaces of measures, De Gruyter Studies in Math., vol. 4, W. de Gruyter, Berlin-New York, 1984.

[DU] J. Diestel and J. J. Uhl, Jr., Vector Measures, Math. Surveys 15, Amer. Math. Soc., Providence, RI, 1977. 
[D1] L. Drewnowski, Topological rings of sets, continuous set function, integration. II, III, Bull. Acad. Polon. Sci. Sér. Sci. Math. Astronom. Phys. 20 (1972), 277-286, 439-445.

[D2] _ Decompositions of set functions, Studia Math. 43 (1973), $23-48$.

[D3] Un théorème sur les opérateurs de $l_{\infty}(\Gamma), \mathrm{C} . \mathrm{R}$. Acad. Sci. Paris Sér. A 281 (1975), 967-969.

[DL] , and I. Labuda, Copies of $c_{0}$ and $\ell_{\infty}$ in topological Riesz spaces, Trans. Amer. Math. Soc. 350 (1998), 3555-3570.

[DW] _ and W. Wnuk, On the modulus of indefinite vector integrals with values in Banach lattices, Atti Sem. Mat. Fis. Univ. Modena 48 (1999), 221-233.

[DS] N. Dunford and J. T. Schwartz, Linear Operators. Part I, Interscience, New York, 1958.

[G] G. Groenewegen, On spaces of Banach lattice valued functions and measures, Thesis, Univ. Nijmegen, Nijmegen, 1982.

[GvR] G. Groenewegen and A. van Rooij, The modulus of a weakly compact operator, Math. Z. 195 (1987), 473-480.

[Ja1] G. J. O. Jameson, Topological M-spaces, Math. Z. 103 (1968), 139150.

[Ja2] Unconditional convergence in partially ordered linear spaces, Math. Ann. 200 (1973), 227-233.

[K] N. J. Kalton, Exhaustive operators and vector measures, Proc. Edinburgh Math. Soc. 19 (1974), 291-300.

[KM-N] G. Kronsbein and P. Meyer-Nieberg, Factorization of vector measures, Arch. Math. 63 (1994), 541-548.

[La] I. Labuda, Completeness type properties of locally solid Riesz spaces, Studia Math. 77 (1984), 349-372.

[Le] D. H. Leung, Lattice absolutely summing operators, J. Operator Theory, 27 (1992), 157-167.

[LT] J. Lindenstrauss and L. Tzafriri, Classical Banach spaces II. Function spaces, Ergebnisse der Mathematik und ihrer Grenzgebiete, vol. 97, Springer-Verlag, Berlin-New York, 1979. 
[O] W. Orlicz, O szeregach doskonale zbieżnych w pewnych przestrzeniach funkcyjnych, Prace Mat. 1 (1955), 393-414 (Polish); English transl., On perfectly convergent series in certain function spaces, W. Orlicz, Collected Papers, Part I, Polish Scientific Publishers, Warszawa, 1988, pp. 830-850.

[R] A. P. Robertson, On unconditional convergence in topological vector spaces, Proc. Roy. Soc. Edinburgh Sect. A 68 (1969), 145-157.

[vR] A. C. M. van Rooij, When do the regular operators between two Riesz spaces form a Riesz space?, Report 8410, Department of Mathematics, Catholic University of Nijmegen, 1984.

[hS] H. H. Schaefer, Banach lattices and positive operators, Springer-Verlag, Berlin-Heidelberg-New York, 1974.

[hSZ] H. H. Schaefer and Xiao-Dong Zhang, A note on order bounded vector measures, Arch. Math. 63 (1994), 152-157.

[kS1] K. D. Schmidt, Decompositions of vector measures in Riesz spaces and Banach lattices, Proc. Edinburgh Math. Soc. 29 (1986), 23-39.

[kS2] _ On the modulus of weakly compact operators and strongly additive vector measures, Proc. Amer. Math. Soc. 102 (1988), 862866.

[kS3] _ Jordan decompositions of generalized vector measures, Pitman Research Notes in Mathematics Series: 214, Longman Scientific \& Technical, Harlow, and J. Wiley \& Sons, New York, 1989.

[S] W. Sierpiński, Sur la convergence absolument uniforme des séries de fonctions, Ganita 1 (1950), 97-101 reprinted in: W. Sierpiński, Oeuvres Choisies, I, PWN - Éditions Sci. Pologne, Warszawa, 1974, pp. 296-300.

[V] A. I. Veksler, Tests for the interval completeness and interval-complete normability of KN-lineals, Izv. Vyš̌. Učebn. Zaved. Matematika 1970, no. 4(95), 36-46. (Russian).

[We] H. Weber, Uniform lattices - I: A generalization of topological Riesz spaces and topological Boolean rings, Ann. Mat. Pura Appl. 160 (1991), 347-370.

[W1] W. Wnuk, On the order-topological properties of the quotient space $L / L_{A}$, Studia Math. 79 (1984), 139-149.

[W2] , Properties of topological Riesz spaces related to vector measures, Atti Sem. Mat. Fis. Univ. Modena 49 (2001), 129-142. 
Faculty of Mathematics and Computer Science,

A. Mickiewicz University

Matejki 48/49, 60-769 Poznań

Poland

E-mail: drewlech@amu.edu.pl

E-mail: wnukwit@amu.edu.pl

Recibido: 3 de Mayo 2001

Revisado: 11 de Enero de 2002 